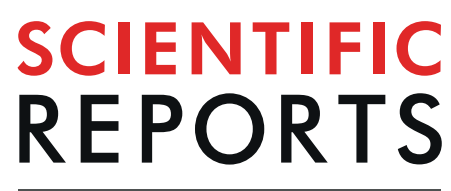

natureresearch

Check for updates

\title{
Origin of $\omega$-phase formation in metastable $\beta$-type Ti-Mo alloys: cluster structure and stacking fault
}

\begin{abstract}
Mingjia Li \& Xiaohua Min ${ }^{\bowtie}$
The $\omega$-phase formation and its collapsed structures in metastable $\beta$-type Ti-Mo alloys were illustrated by first-principles calculations and experimental evidence of a partially collapsed $\omega$-phase in the nanoscale Mo-depleted region under a rapid cooling via high-angle annular dark-field scanning transmission electron microscopy. The ease of $\omega$-phase formation within -Mo-Ti-Mo- poor cluster structure was not only due to the low energy barrier in the collapse pathway, which was caused by the reduced lattice distortion, but also due to the softening of the shear modulus $\left(G_{111}\right)$ as a result of the small charge density difference. The most stable collapsed structure of the $\omega$-phase strongly depended on the minimum stacking fault energy among different collapse degrees in accordance to the smallest charge density difference. Therefore, the concurrent compositional and structural instabilities of the $\omega$-phase was attributed to the coupling effect of the cluster structure with stacking fault from the atomic and electronic basis.
\end{abstract}

Omega $(\omega)$ phase was firstly identified in Beta ( $\beta$ ) type Ti-Cr alloys by Frost et al. in $1954^{1}$. Since then, the $\omega$-phase has been observed in numerous group IV transition metals (Ti, Ta, Hf and $\mathrm{Zr}$ ) and their alloys containing appropriate $\beta$-stabilizing elements ${ }^{2-4}$. According to the orientation relationship unanimously described as $[0001]_{\omega} / /$ $[111]_{\beta}$ and $(11 \overline{2} 0)_{\omega} / /(01 \overline{1})_{\beta}$, four crystallographic $\omega$-variants can be found in the $\beta$-matrix ${ }^{5}$. The $\omega$-phase can be classified into the deformation-induced $\omega$-phase, athermal $\omega$-phase, and isothermal $\omega$-phase based on its formation process. The morphologies of the $\omega$-phase can be plate-like $e^{6-8}$ and ellipsoidal or cuboidal ${ }^{9-11}$, while its sizes range from several to dozens of nanometers depending on both the stressing condition and the compositional partitioning ${ }^{12-15}$. The athermal $\omega$-phase is present in alloys during rapid cooling from the high-temperature single $\beta$-phase field ${ }^{10,11}$. Previous works have extensively investigated the effect of $\omega$-phase formation on mechanical and functional properties of metastable $\beta$-type titanium alloys ${ }^{16-21}$. As a metastable phase, the $\omega$-phase has attracted the attention of researchers owing not only to its effects on the above properties but also to its complex formation mechanism.

Fontaine et al. ${ }^{22}$ reported that the $\omega$-phase can be obtained by submitting the body-centered cubic (bcc) lattice to a 2/3(111) longitudinal displacement wave. They also performed a Monte Carlo simulation to demonstrate that the $\omega$-phase transformation was a product of the linear displacive defects along the $[111]_{\beta}$ direction. By performing frozen phonon calculations, Ho et al. ${ }^{23}$ found that the formation of an ideal $\omega$ structure from the $\beta$-phase was attributed to the reduction of ground state energy for a $2 / 3<111\rangle_{\beta}$ phonon. Lin $e t$ al..$^{24}$ demonstrated that the athermal $\omega$-phase formation was caused by an atom collapse from the $(110)_{3}$ plane to the $(111)_{\beta}$ plane along the $<111\rangle_{\beta}$ direction. Although different mechanisms have been proposed to describe the $\omega$-phase formation, the $\omega$-phase has been widely agreed to form crystallographically via a collapse mechanism that involves various degrees of alternate pairs of $\{111\}_{\beta}$ planes to an intermediate position ${ }^{25}$. The collapse degree is evaluated by shear magnitude $(z)$, which refers to the atomic moving distance on the $(112)_{3}$ plane along the $[11 \overline{1}]_{3}$ direction ${ }^{26-31}$. When the collapse is completed, the $\omega$-phase shows a hexagonal structure $(z=1 / 6)$, whereas a partial collapse leads to a trigonal structure $(z<1 / 6)$. However, the structural evolution of the $\omega$-phase from trigonal to hexagonal remains under discussion.

When the $\beta$-phase stability is low in metastable $\beta$-type titanium alloys, the collapse degree is close to $1 / 6$ but is less than $1 / 6$ or even equal to 0 when the stability is relatively or sufficiently high. Hickman ${ }^{32}$ suggested that the $\omega$-phase formed in the solute poor region because of compositional partitioning due to phase separation in $\mathrm{Zr}-\mathrm{Nb}$ alloys. Liu et al. ${ }^{6}$ reported that trigonal and hexagonal $\omega$-phases were simultaneously present due to the 
inhomogeneity of composition and defect in a water-quenched Ti-9Cr-0.2 $\mathrm{O}$ alloy (all contents are expressed as wt.\% without special instructions). By coupling aberration-corrected high-angle annular dark-field (HAADF) with the three-dimensional atom tomography (APT) technique, Banerjee et al. ${ }^{10,33}$ recently presented direct experimental evidence of phase separation in a rapid-cooling Ti-18Mo alloy and the formation of a partially collapsed $\omega$-phase in Mo-depleted region. Although experimental evidence indicates that the $\omega$-phase formation and its structural evolution strongly depend on alloying element content, the physical nature of the $\omega$-phase formation under poor solute content and its effects on the collapsed structure remain unclear.

Issues related to atomic and electronic levels have been extensively studied in the literature by using first-principles calculations. Lai et al. ${ }^{34}$ calculated the energetic pathway of the $\omega$-phase formation in Ti-Nb alloys by shifting the atoms on the $\{112\}_{\beta}$ plane along the $\left.<111\right\rangle_{\beta}$ direction and found that the energy barrier in the pathway depended on the composition. Niu et al. ${ }^{35}$ evaluated the effect of $\mathrm{O}$ element on the $\beta$ to $\omega$ transformation in $\mathrm{Ti}_{3} \mathrm{Nb}$ and found that such element increased the energy barrier for the $\beta$ to $\omega$ transformation and the formation energy of the $\omega$-phase. Choudhuri et al. ${ }^{11}$ calculated the charge density difference in the supercells of Ti-V alloys and found that $\omega$-phase formation with high $\mathrm{V}$ content was suppressed by the strong $\mathrm{V}-\mathrm{V}$ bond. Banerjee et al. ${ }^{33}$ simulated the $\omega$-phase transformation via the collapse mechanism in Ti-Mo alloys and found that a partially collapsed structure was energetically favored over a fully collapsed structure with an Mo content of 8.33 at.\%. The abovementioned first-principles calculations were performed by constructing supercells with an uniform or ordered distribution of solute atoms. However, the aforementioned experimental evidence obtained via high resolution electron microscopy and atom probe microanalysis reveals that composition partitioning is ubiquitous in metastable titanium alloys. Alloying elements with cluster structures have been successfully utilized to describe the phase structures in $\mathrm{Al}-\mathrm{Zr}^{36}, \mathrm{Au}_{-} \mathrm{Pt}^{37}$, and $\mathrm{Ti}^{-} \mathrm{V}^{38,39}$. In our previous work ${ }^{40,41}$, the -Mo-Ti-Mo- cluster structure was constructed in the supercells of $\beta$-phase, $\alpha^{\prime \prime}$-martensite and $\omega$-phase in Ti-Mo alloys. The formation sequence of $\alpha^{\prime \prime}$-martensite and $\omega$-phase is attributed to the competition among several moduli along specific directions, including tetragonal shear elastic constant $\left(C^{\prime}\right)$, Young's modulus $\left(E_{100}\right)$, and shear modulus $\left(G_{111}\right)$. However, given that only the -Mo-Ti-Mo- cluster structure was considered in our previous work, the $\omega$-phase with a collapsed structure easily formed under a poor solute content is not yet comprehensively understood.

Generally, the collapse mechanism of the $\omega$-phase changes the "ABCABCA" sequence to "ABABA" on the $\{112\}_{\beta}$ plane along the $\langle 111\rangle_{\beta}$ direction $^{34,42,43}$. On the basis of the reported correlation between the $\omega$-phase and $\{112\}_{\beta}<111>_{\beta}$ twins in Ti-Nb alloys ${ }^{34,44}$, the structural evolution of the $\omega$-phase can be influenced by the stacking fault on the $\{112\}_{3}$ plane. This issue needs to be solved by focusing on the interaction between cluster structure and stacking fault. The stacking fault energy in face-centered cubic ( $\mathrm{fcc}$ ) alloys has been widely used in evaluating phase stability and deformation mode ${ }^{45-52}$. However, only few studies have examined the stacking fault energy in metastable $\beta$-type titanium alloys. Wu et al. ${ }^{43}$ used coherent potential approximation to construct a model in $\mathrm{Ti}-30 \mathrm{Nb}-3 \mathrm{Pd}$ alloy with stacking fault and calculated the energy change during the $\omega$-phase transformation to evaluate the $\omega$-phase structure. By considering the aforementioned nano-scale composition partitioning in metastable titanium alloys and the results of our previous work, the coupling effect of the cluster structure and stacking fault may help reveal the origin of $\omega$-phase formation.

By constructing supercells with cluster structure and stacking fault in metastable Ti-Mo alloys, the stacking fault and formation energies obtained via first-principles calculations were used to evaluate the $\omega$-phase formation and its collapsed structure. Experimental evidence of the nano-scale compositional and structural instabilities of the $\omega$-phase was obtained via aberration-corrected high-resolution scanning transmission electron microscopy in a water-quenched Ti-Mo alloy. The ease of $\omega$-phase formation in the -Mo-Ti-Mo-cluster poor region was mainly due to the softening effect of the shear modulus, whereas its collapse degree could be attributed to the presence of minimum stacking fault energy, which was further discussed in terms of the calculated lattice distortion and change density difference from the atomic and electronic basis.

\section{Results}

Fig. 1(a-f) shows the constructed supercells of the $(1 \overline{1} 0)_{3}$ plane with cluster structure and stacking fault. The $\{111\}_{\beta}$ planes in the bcc lattice were denoted by "ABCABCA" $\left(z=0\right.$, i.e., $\beta$-phase) in Fig. $1(\mathrm{a}, \mathrm{d})$. The adjacent $(11 \overline{2})_{\beta}$ planes at positions -1 and $-2\left(\mathrm{P}_{-1}\right.$ and $\left.\mathrm{P}_{-2}\right)$ sheared toward each other, thereby changing " $\mathrm{AB}^{\prime} \mathrm{C}^{\prime} \mathrm{AB}^{\prime} \mathrm{C}^{\prime} \mathrm{A}^{\text {" }}(z<1 / 6$, i.e., partially collapsed or trigonal structure) in Fig. $1(\mathrm{~b}, \mathrm{e})$ to " $\mathrm{AB}^{\prime} \mathrm{AB}^{\prime} \mathrm{A}^{\prime}(z=1 / 6$, i.e., fully collapsed or hexagonal structure) in Fig. 1(c,f). Fig. $1(\mathrm{a}-\mathrm{c})$ shows the -Mo-Ti-Mo- linear unit along the $[11 \overline{1}]_{3}$ direction in the $2 \mathrm{Mo}$ supercell. The presence of seven positions from $\mathrm{P}_{-3}$ to $\mathrm{P}_{3}$ indicated that the cluster moved away from the stacking fault. These cluster positions were labeled by the Ti atom in the middle of the linear unit (e.g., the two cluster positions at $\mathrm{P}_{-3}$ and $\mathrm{P}_{2}$ in the figure). Fig. 1(d-f) shows the trigonal cluster comprising -Mo-Ti-Mo- linear units along the $[1 \overline{1} 1]_{3},[\overline{1} 11]_{3}$, and $[001]_{\beta}$ directions (in side view) in the $3 \mathrm{Mo}$ supercell. Four cluster positions from $\mathrm{P}_{-3}$ to $\mathrm{P}_{3}$, such as the two cluster positions at $\mathrm{P}_{-3}$ and $\mathrm{P}_{1}$ that were labeled by the Mo atom in the middle of the cluster (the left Mo atom in side view), described the position correlation between cluster structure and stacking fault.

Fig. 2(a,b) shows stacking fault energy as a function of collapse degree in the constructed 2Mo and 3Mo supercells with different cluster positions. In the initial bcc lattice, no stacking fault energy was observed for all linear and trigonal cluster positions. Fig. 2(a) shows that the stacking fault energy decreases along with collapse degree for each cluster position in the 2Mo supercell. A minimum value $\left(\gamma_{S F, \min }^{z}\right)$ was observed in each curve as denoted by the circle, thereby suggesting that the corresponding collapsed structure of the $\omega$-phase was the most stable among all collapse degrees. With the linear cluster moving away from the stacking fault from $\mathrm{P}_{-3}$ to $\mathrm{P}_{3}$, the stacking fault energy decreased, whereas the collapse degree at minimum stacking fault energy increased from 6.2/48 at $\mathrm{P}_{-3}$ to $8 / 48$ at $\mathrm{P}_{2}$ and $\mathrm{P}_{3}$. In the $3 \mathrm{Mo}$ supercell with the cluster at $\mathrm{P}_{-3}$ as shown in Fig. $2(\mathrm{~b})$, the stacking fault energy increased along with the collapse degree and its minimum value was zero, thereby suggesting that the bcc lattice was the most stable structure without collapse. Similarly, the stacking fault energy decreased along with 

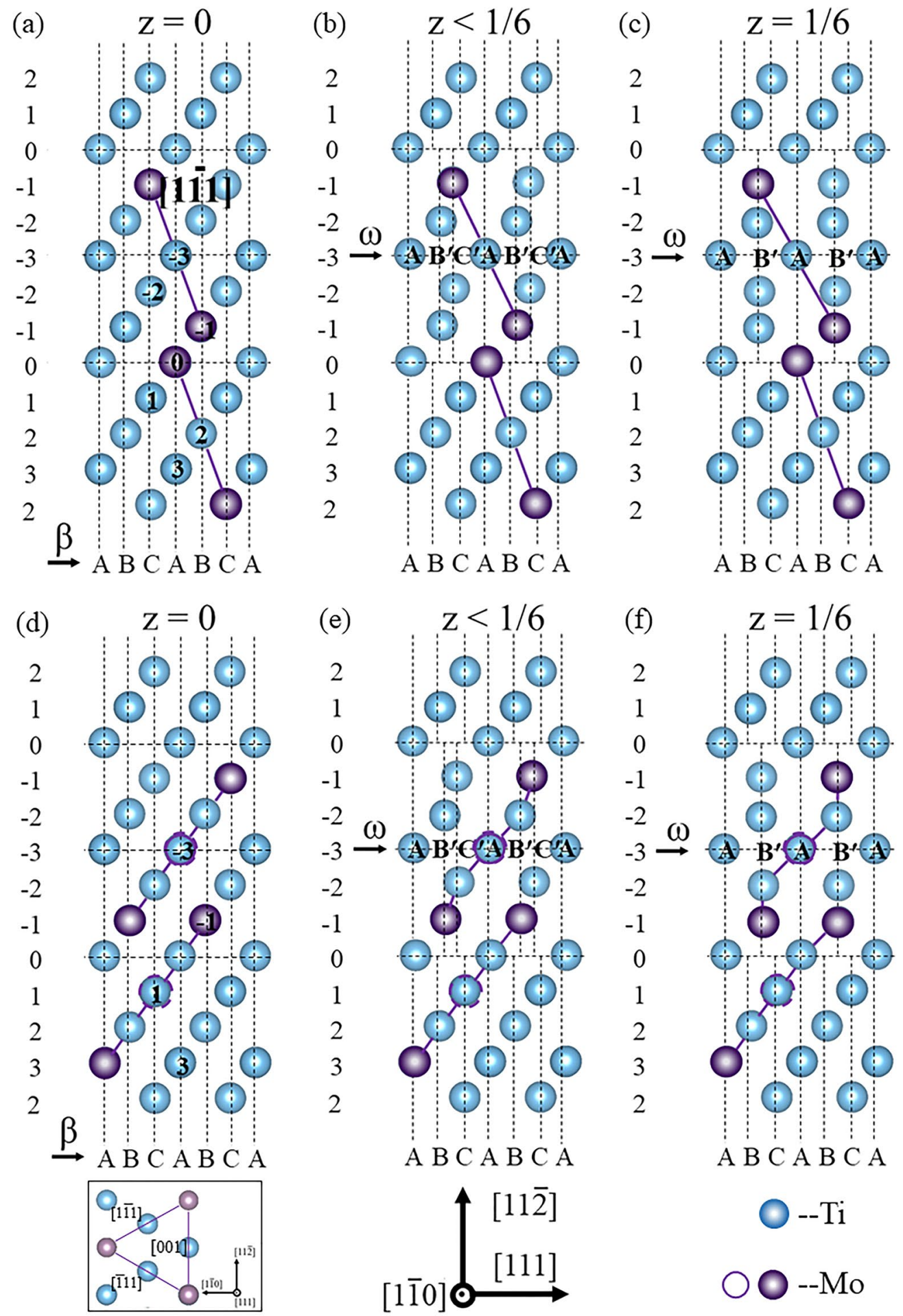

Figure 1. Constructed supercells on $(1 \overline{1} 0)_{\beta}$ planes with cluster structure and stacking fault. (a-c) 2 Mo supercell with -Mo-Ti-Mo- linear cluster at two positions $\left(\mathrm{P}_{-3}\right.$ and $\left.\mathrm{P}_{2}\right)$ for example; $(\mathbf{d}-\mathbf{f}) 3 \mathrm{Mo}$ supercell with -MoTi-Mo- trigonal cluster at two positions $\left(\mathrm{P}_{-3}\right.$ and $\left.\mathrm{P}_{1}\right)$ for example; $(\mathbf{a}, \mathbf{d})$ initial $\beta$-phase $(z=0)$ with the $\left.{ }_{(111}\right)_{\beta}$ planes labeled by "ABCA;" (b,e) $(111)_{3}$ planes with partial stacking fault $(z<1 / 6)$ labeled by "AB' $\mathrm{C}^{\prime} \mathrm{A}$;" and (c,f)

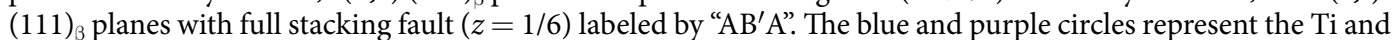
Mo atoms, respectively. The open and solid circles of the Mo atoms in the $3 \mathrm{Mo}$ supercell were located at two adjacent $(1 \overline{1} 0)_{\beta}$ planes.

collapse degree when the trigonal cluster was located far from the stacking fault, and the collapse degree at minimum value increased from $6 / 48$ at $\mathrm{P}_{-1}$ to $8 / 48$ at $\mathrm{P}_{3}$. To further evaluate the deviation of collapse degree from the trigonal to hexagonal structure of the $\omega$-phase, the difference of stacking fault energy $\left(\Delta \gamma_{\mathrm{SF}}^{z}\right)$ between the stable 

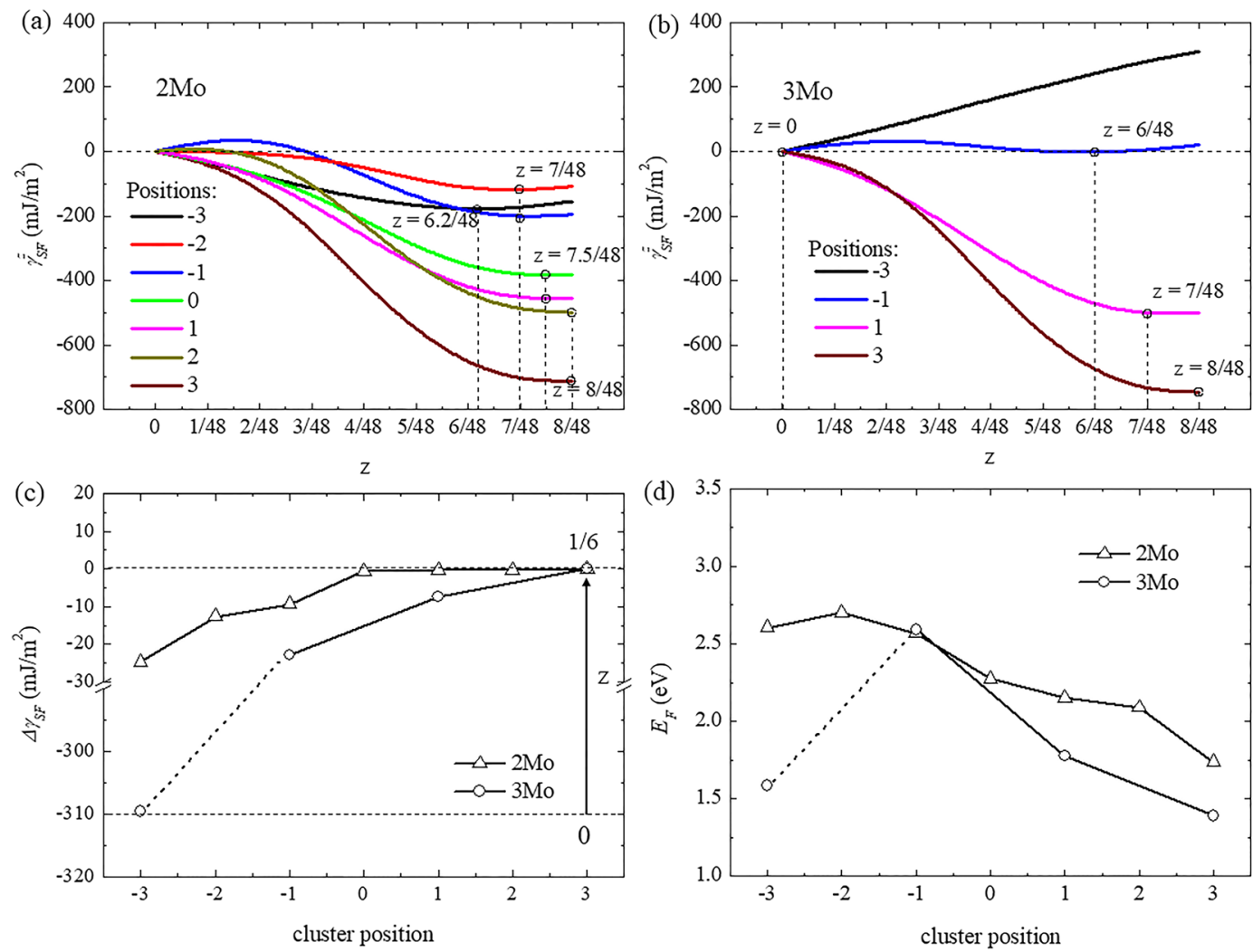

Figure 2. Stacking fault energy $\left(\gamma_{S F}^{z}\right)$ as a function of collapse degree $(z)$ in (a) 2Mo supercell and (b) 3Mo supercell with different cluster positions. The minimum stacking fault energy corresponding to collapse degree was denoted by the circle in each curve. (c) Difference in stacking fault energy $\left(\Delta \gamma_{S F}\right)$ between a stable structure with minimum stacking fault energy and a fully collapsed structure, and (d) formation energy $\left(E_{F}\right)$ of a stable structure with minimum stacking fault energy as a function of cluster position in $2 \mathrm{Mo}$ and $3 \mathrm{Mo}$ supercells.

structure and the fully collapsed structure was obtained as a function of cluster position as shown in Fig. 2(c). The lower $\Delta \gamma_{S F}^{z}$ indicated that the stable structure of the $\omega$-phase exhibited a smaller collapse degree. In the $2 \mathrm{Mo}$ supercell, $\Delta \gamma_{S F}^{z}$ had the minimum value of $-24.7 \mathrm{~mJ} / \mathrm{m}^{2}$ with a liner cluster at $\mathrm{P}_{-3}$, thereby suggesting that the stable trigonal structure had the smallest collapse degree (6.2/48). With the cluster moving away from the stacking fault, the $\Delta \gamma_{S F}^{z}$ increased to zero at $\mathrm{P}_{2}$ and $\mathrm{P}_{3}$ corresponding to the stable hexagonal structure. The $\Delta \gamma_{S F}^{z}$ in the 3 Mo supercell exhibited a similarly increasing trend with the trigonal cluster moving away from the stacking fault but was lower than that in the 2Mo supercell at the same position, thereby reducing the collapse degree of the $\omega$-phase, especially at $\mathrm{P}_{-3}$ (bcc lattice) with the lowest value of $-310 \mathrm{~mJ} / \mathrm{m}^{2}$. Fig. 2 (d) shows the formation energy $\left(E_{F}\right)$ of the stable structure with minimum stacking fault energy as a function of cluster position in $2 \mathrm{Mo}$ and $3 \mathrm{Mo}$ supercells. A lower $E_{F}$ indicated that the cluster structure was more stable in the position of the supercell. The $E_{F}$ decreased from $2.5 \mathrm{eV}$ to $2.1 \mathrm{eV}$ in the $2 \mathrm{Mo}$ supercell from $\mathrm{P}_{-3}$ to $\mathrm{P}_{3}$ and from $2.5 \mathrm{eV}$ to $1.4 \mathrm{eV}$ in the $3 \mathrm{Mo}$ supercell from $\mathrm{P}_{-1}$ to $\mathrm{P}_{3}$. However, the $E_{F}$ in the $3 \mathrm{Mo}$ supercell at $\mathrm{P}_{-3}$ exhibited a low value of $1.6 \mathrm{eV}$ due to the stable bcc lattice. Consequently, the -Mo-Ti-Mo- cluster structures should occupy positions far from the stacking fault, and the stable collapsed structure of the $\omega$-phase changed from trigonal to hexagonal due to the low formation and stacking fault energies.

The TEM dark-field image and its selected area electron diffraction (SAED) pattern were obtained in a water-quenched sample of Ti-15Mo alloy. As shown by the arrows in Fig. 3(a), the black area is mainly $\beta$-matrix which may contain a small amount of $\omega$-particles, while the white particles are mainly $\omega$-phase with a diameter of less than $10 \mathrm{~nm}$. The SAED pattern in Fig. 3(b) exhibited three sets of spots along the $[110]_{\beta}$ zone axis that corresponded to two $\omega$-phase variants of $\omega_{1}$ and $\omega_{2}$ (denoted by the red and green squares, respectively) as well as the $\beta$-phase (denoted by the blue square). The diffraction spots selected to produce the dark-field image was marked by a white circle in Fig. $3(\mathrm{~b})$. The intensity profiles of two $\omega$-variants along the $\langle 111\rangle_{\beta}$ direction were denoted by the dotted lines and were analyzed on the basis of the SAED pattern in Fig. 3(c). The peak positions of $\omega$-phase spots slightly deviated from relative displacements of $1 / 3$ and $2 / 3$, suggesting the formation of the $\omega$-phase without an ideal hexagonal structure. To quantitatively evaluate its collapse degree, the ratio of $d_{0002 \omega}^{*} / d_{222 \beta}^{*}$ in reciprocal space was obtained by establishing a formula with collapse degree as derived from the Appendix of our previous work ${ }^{41}$. As a result, the $\omega_{1}$-variant and $\omega_{2}$-variant had collapse degrees of $0.164 \pm 0.001$ and $0.163 \pm 0.001$, respectively. In terms of the orientation relationship between the $\beta$-phase and $\omega$-phase and the structural evolution of the $\omega$-phase as shown in Fig. 3(d), the $\omega$-phase with a partially collapsed structure was formed in the alloy. 


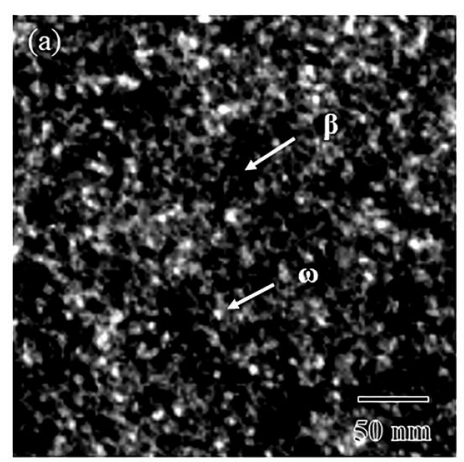

(d)

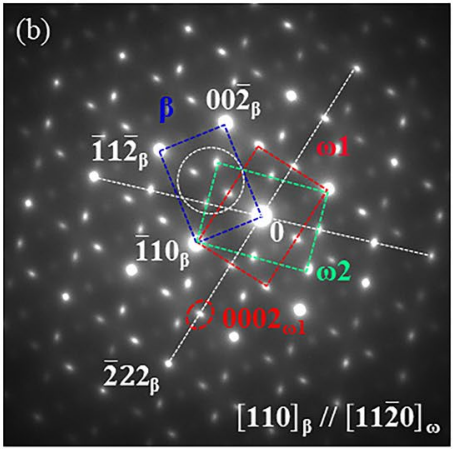

$\mathbf{a}_{\omega}=\sqrt{ } \mathbf{2} \mathbf{a}_{\beta}$

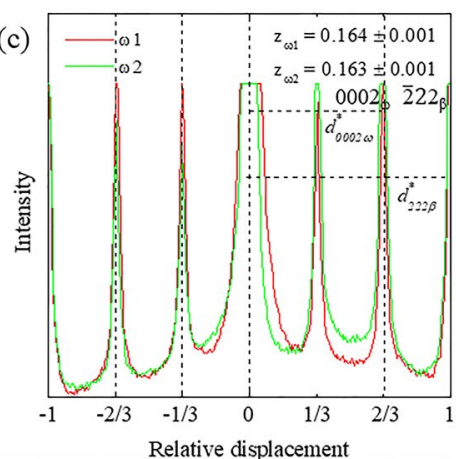

$[\overline{1} 11]_{\beta} / /[0001]_{\omega}$
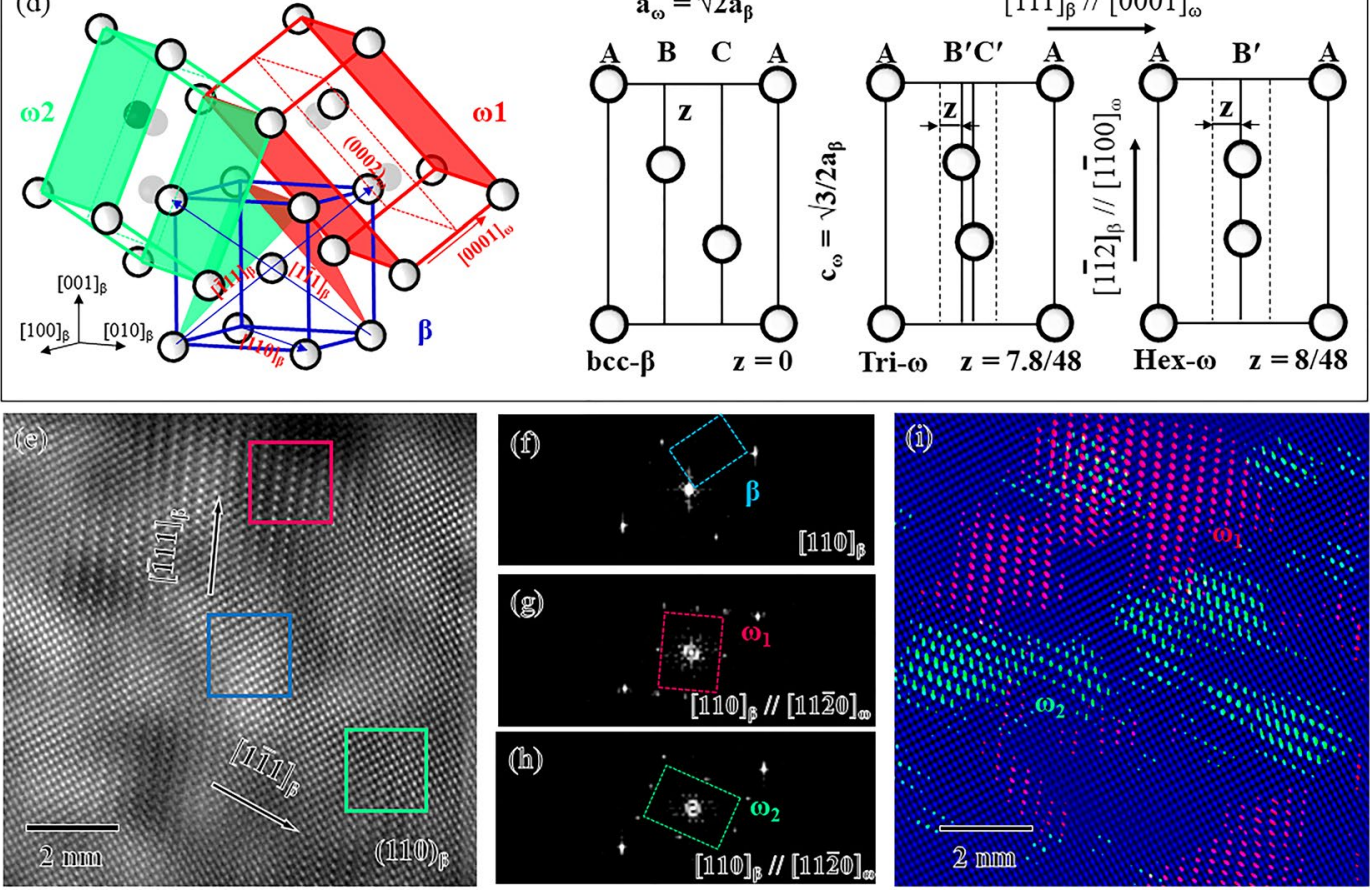

Figure 3. (a) TEM dark-field image and (b) selected area diffraction pattern of a water-quenched sample in Ti$15 \mathrm{Mo}$ alloy; (c) intensity profiles of diffraction spots along white lines as shown in (b); and (d) schematic of the orientation relationships between $\beta$-phase and two $\omega$-variations and the structural evolution of the $\omega$-phase. (e) Fourier filtered HAADF-STEM; FFT patterns of the (f) $\beta$-phase, $(\mathbf{g}) \omega_{1}$-variant, $(\mathbf{h}) \omega_{2}$-variant corresponding to regions denoted by the blue, red, and yellow squares in (e), respectively, and (i) overlay of RGB images filtered by using reflection spots from the $\omega_{1}$-variant (R: red), $\omega_{2}$-variant (G: green), and $\beta$-phase (B: blue). The zone axis is parallel to the $[110]_{\beta}$ direction.

The Fourier filtered image in Fig. 3(e) exhibited nano-scale regions with relatively brighter and darker contrasts, which indicated compositional partitioning within the $\beta$-matrix. The bright and dark regions corresponded to the Mo-enriched and Mo-depleted regions, respectively. The typical structural features within different regions were further detected by the FFT patterns. Only the $\beta$-phase spots in Fig. 3(f) were obtained within the Mo-enriched region (denoted by the blue square in Fig. 3(e)). The two $\omega$-variants $\left(\omega_{1}\right.$ and $\left.\omega_{2}\right)$ spots in Fig. 3(g,h) were present in the Mo-depleted regions (denoted by the red and green squares in Fig. 3(e), respectively). The overlay of RGB images was filtered by using reflection spots from the $\omega_{1}$-variant (R: red), $\omega_{2}$-variant (G: green), and $\beta$-phase (B: blue), which corresponded to the Mo-depleted and Mo-enriched regions in Fig. 3(e). The green and red regions indicated that the formed $\omega$-phase had a size of several nanometers. These experimental results indicated that the $\omega$-phase with a trigonal structure was prone to form in the nano-scale Mo-depleted region due to compositional partitioning.

Fig. 4(a) shows the Fourier filtered HAADF-STEM image of a water-quenched sample of Ti-15Mo alloy along the $[110]_{\beta}$ zone axis. The $\omega$-phase and $\beta$-matrix were distinguished by the yellow dotted lines from the bottom to the top of the image. Three enlarged images (Fig. 4(b-d)) corresponding to the three regions denoted by the red squares in Fig. 4(a) indicated the different collapse degrees of the $\{111\}_{3}$ plane. The $\beta$-matrix in Fig. 4(b) exhibited no collapse in the Mo-enriched region, whereas the $\omega$-phase with partial collapse in Fig. 10(c,d) was close to the boundary of the $\beta$-matrix and in the Mo-depleted region. To quantitatively evaluate the collapse degree, a plot of atomic column intensity as a function of distance was obtained along the $\langle 111\rangle_{\beta}$ direction, and the relative 

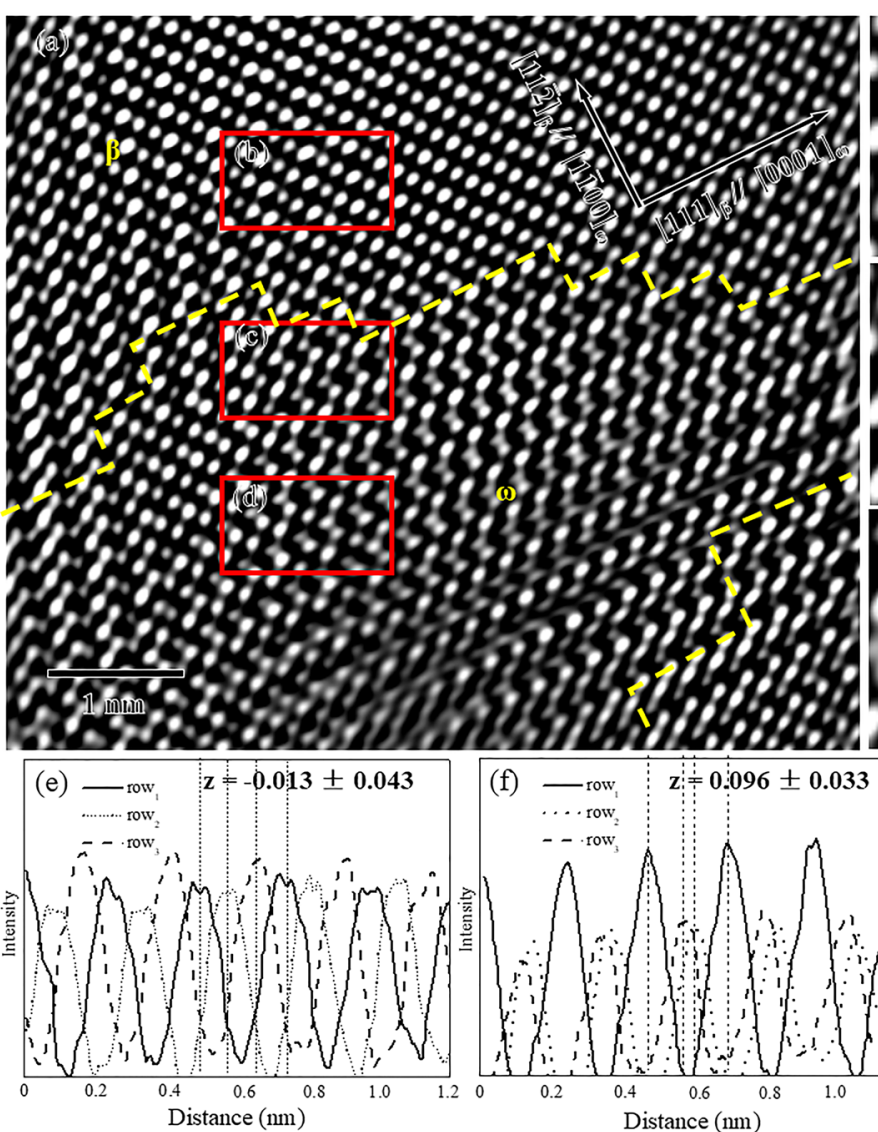

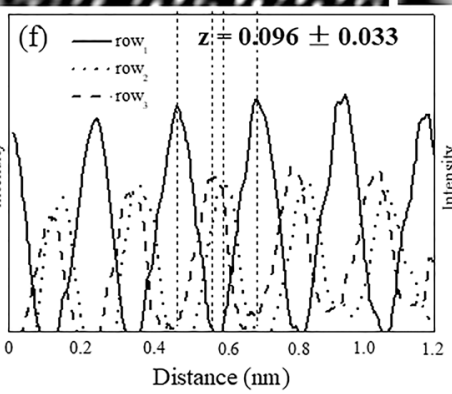

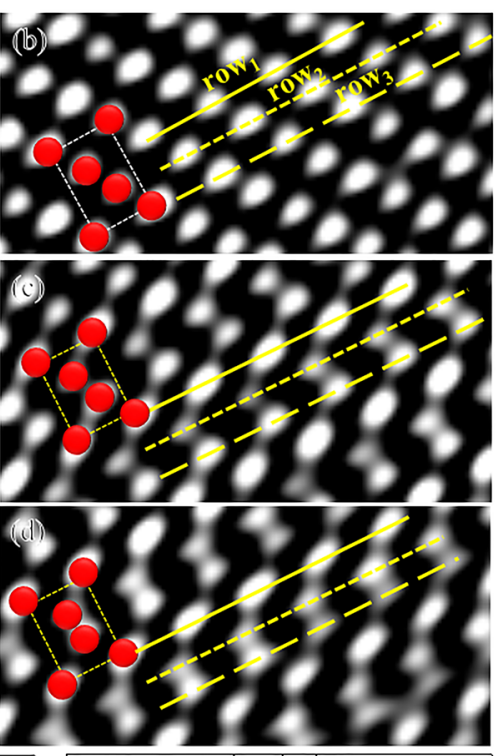

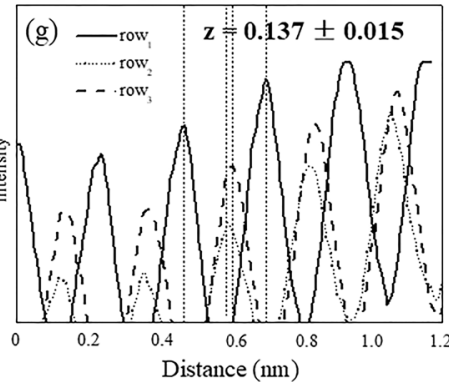

Figure 4. (a) Fourier filtered HAADF-STEM image along the $\langle 110\rangle_{\beta}$ zone axis with the $\omega$-phase and $\beta$-matrix distinguished by yellow dotted lines. The enlarged images are indicated by red squares of (a) with yellow row , $_{1}$ row $_{2}$ and row 3 along $[111]_{\beta}$ direction and red motifs. (b) in Mo-enriched region, (c) near boundary of $\beta$-matrix, and (d) in Mo-depleted region. (e-g) The intensity profiles of the three rows and the collapse degrees $(z)$ corresponded to $(\mathbf{b})$ to $(\mathbf{d})$, respectively.

positions of peaks were denoted by the dashed lines. The value of $z$ was computed as the ratio of distance that the $(11 \overline{2})_{3}$ plane moves along the $[111]_{\beta}$ direction to $1 / 2<111>\mathrm{a}_{3}$. In the Mo-enriched region, the peaks of row $_{2}$ and

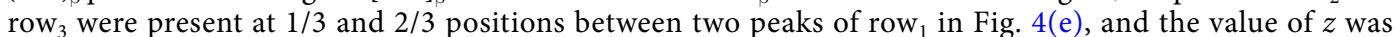
$-0.013 \pm 0.043$. Near the boundary of the $\beta$-matrix, the peaks of row ${ }_{2}$ and row $_{3}$ were closed due to the $\langle 111\rangle_{\beta}$ atomic collapse as shown in Fig. 4(f), and the value of $z$ was $0.096 \pm 0.033$. In the Mo-depleted region, the value of $z$ further increased to $0.137 \pm 0.015$ as shown in Fig. $4(\mathrm{~g})$. Therefore, the collapse degree of the $\omega$-phase continuously increased from the Mo-enriched region to the Mo-depleted region.

\section{Discussion}

The $\omega$-phase that was easily formed in the -Mo-Ti-Mo- cluster poor region and its collapsed structure strongly depended on the present minimum stacking fault energy obtained via theoretic calculations. Moreover, the partially collapsed $\omega$-phase was most likely present in the nano-scale Mo-depleted region according to the results of experimental observations. From the atomic and electronic basis, both lattice distortion and change density difference were calculated to study the reasons behind the $\omega$-phase formation via the cluster structure and its collapsed structural evolution combined with stacking fault. The concurrent compositional and structural instabilities of the $\omega$-phase was also discussed from the view of a metastable "frozen" state of Mo atoms due to the rapid cooling condition.

$\omega$-phase formation by cluster structure. On the basis of the aforementioned collapse mechanism of the $\omega$-phase, two factors were identified to influence the collapse process on the $\{112\}_{\beta}$ plane along the $\left\langle 111>_{\beta}\right.$ direction: one is the extrinsic energy barrier in the pathway, whereas the other is the intrinsic resistance of shear along a specific direction. The energy barrier is affected by the lattice distortion, which suppresses the displacive phase transformation $^{53}$. The lattice distortion in this study was caused by the difference in radius between $\mathrm{Mo}$ and $\mathrm{Ti}$ atoms, thereby resulting in that the $(11 \overline{2})_{3}$ plane was not flat with up-and-down hills as shown in Fig. $5(\mathrm{a}, \mathrm{b})$ to supply the energy barriers in the pathway of $[111]_{\beta}$ direction. In $2 \mathrm{Mo}$ and $3 \mathrm{Mo}$ supercells, the up-and-down hills in a certain region as denoted by the red dotted lines (i.e., lattice distortion) decreased along with the position of the cluster structure from $\mathrm{P}_{-3}$ to $\mathrm{P}_{3}$. The trigonal cluster structure in the $3 \mathrm{Mo}$ supercell caused a more serious lattice distortion than that of the linear cluster structure in the $2 \mathrm{Mo}$ supercell. To quantitatively evaluate the effect 

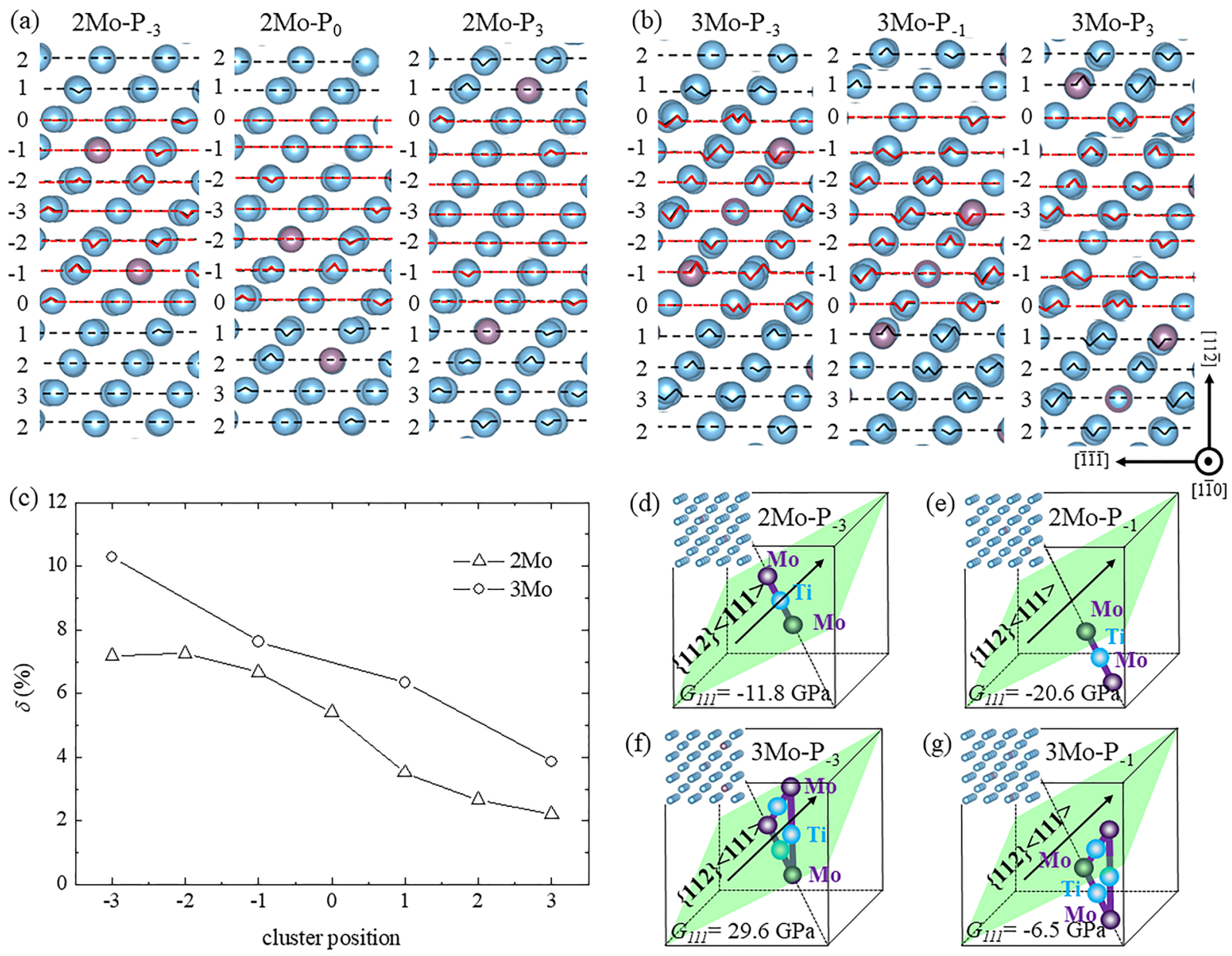

(d)

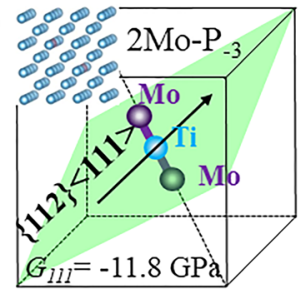

(f)

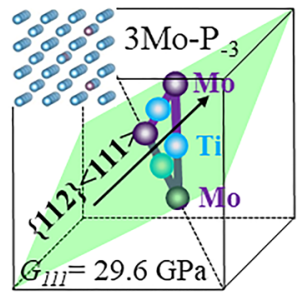

(e)

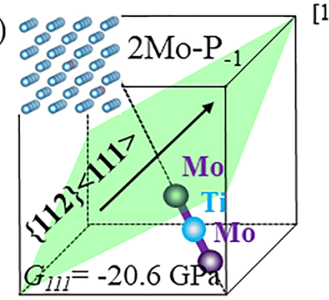

(g)

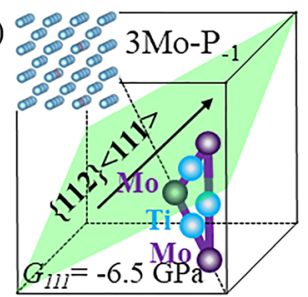

Figure 5. Lattice distortion indicated by up-and-down hills with (a) linear cluster positions of $\mathrm{P}_{-3}, \mathrm{P}_{0}$, and $\mathrm{P}_{3}$ in the 2Mo supercell; (b) trigonal cluster positions of $\mathrm{P}_{-3}, \mathrm{P}_{-1}$, and $\mathrm{P}_{3}$ in the $3 \mathrm{Mo}$ supercell; and (c) change in the lattice distortion $(\delta)$ of the $[11 \overline{2}]_{3}$ direction, with the position of cluster structures in the region of supercells denoted by red dotted lines. Schematic of the effect of cluster position on shear modulus $\left(G_{111}\right)$ with the constructed $2 \mathrm{Mo}$ and $3 \mathrm{Mo}$ supercells. (d) Linear cluster at $\mathrm{P}_{-3} ;(\mathbf{e})$ linear cluster at $\mathrm{P}_{-1} ;(\mathbf{f})$ trigonal cluster at $\mathrm{P}_{-3}$; and (g) trigonal cluster at $\mathrm{P}_{-1}$.

of cluster structures, the lattice distortion of the $[11 \overline{2}]_{3}$ direction $(\delta)$ was obtained as shown in Fig. 5(c). The value of $\delta$ decreased from $7.2 \%$ to $2.2 \%$ in the 2 Mo supercell and from $10.3 \%$ to $3.8 \%$ in the 3 Mo supercell along with the cluster position from $\mathrm{P}_{-3}$ to $\mathrm{P}_{3}$, respectively. In other words, the extrinsic energy barrier for the collapse process of the $\omega$-phase was reduced within -Mo-Ti-Mo- poor region, thereby promoting the formation of this phase.

Shear resistance is reflected by the shear modulus along a certain direction, and its softening effect can benefit the displacive phase transformation. For example, the softening of tetragonal shear elastic constant $\left(C^{\prime}\right)$ with respect to the shear resistance on the $\{110\}_{3}$ plane along the $\langle 110\rangle_{\beta}$ direction enhanced the $\alpha^{\prime \prime}$-martensite formation in titanium alloys ${ }^{41,54,55}$. Meanwhile, shear modulus $\left(G_{111}\right)$ (i.e., shear resistance on the $\{112\}_{\beta}$ plane along the $\langle 111\rangle_{\beta}$ direction) was associated with the collapse mechanism of the $\omega$-phase. Our previous work ${ }^{41}$ reported that the softening of $G_{111}$ could benefit the $\omega$-phase formation and strongly depended on the Mo content in metastable Ti-Mo alloys. Therefore, $G_{111}$ was reduced when the cluster structure moved away from the initial position of $\mathrm{P}_{-3}$ in Fig. 1(a,d) and softened much easier with the linear cluster than with the trigonal cluster. To further illustrate the correlation between cluster structure and shear modulus, the supercells containing 54 atoms with -Mo-Ti-Mo- linear and trigonal clusters were used to calculate $G_{111}$, which can be derived by the elastic constants of the bcc structure ${ }^{6,41}$. Given the size limitations of the constructed supercells, only the two typical cluster positions of $\mathrm{P}_{-3}$ and $\mathrm{P}_{-1}$ were considered in this study as shown in Fig. $5(\mathrm{~d}-\mathrm{g})$. The value of $G_{111}$ decreased from $-11.8 \mathrm{GPa}$ to $-20.6 \mathrm{GPa}$ in Fig. 5(d,e) and from 29.6 GPa to -6.5 GPa in Fig. 5(f,g) along with the shifting of cluster position from $\mathrm{P}_{-3}$ and $\mathrm{P}_{-1}$ in $2 \mathrm{Mo}$ and $3 \mathrm{Mo}$ supercells, respectively. Therefore, the intrinsic shear resistance for the collapse process was softened within the -Mo-Ti-Mo- cluster poor region.

Shear modulus is also associated with the atomic bonding in adjacent planes, which can be evaluated based on the charge density difference. A larger charge density difference corresponds to a stronger atomic bonding ${ }^{11}$. Gao et al. ${ }^{56}$ reported that the charge density difference on the (110) plane along the $\langle 111\rangle_{\beta}$ direction was high for pure $\mathrm{Cr}$ but gradually decreased along with the addition of $\mathrm{V}$, thereby reducing $G_{111}$. Here, the shear modulus 
(a) $2 \mathrm{Mo}-\mathrm{P}_{-3}$

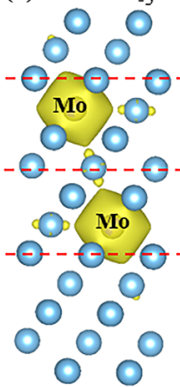

(b) $2 \mathrm{Mo}-\mathrm{P}_{0}$

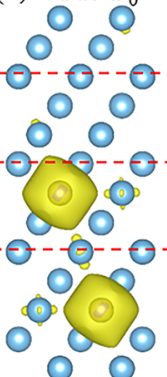

(c) $2 \mathrm{Mo}-\mathrm{P}_{3}$

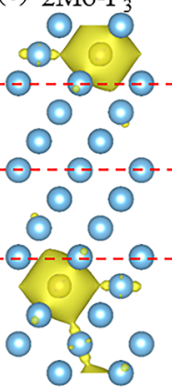

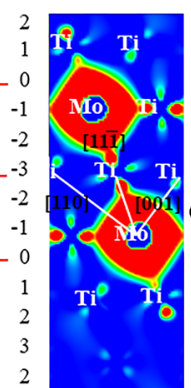

(d) $3 \mathrm{Mo}_{-3}$

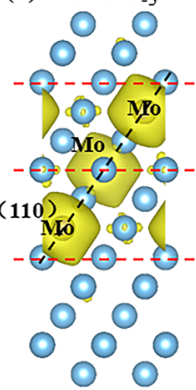

(e) $3 \mathrm{Mo}-\mathrm{P}$
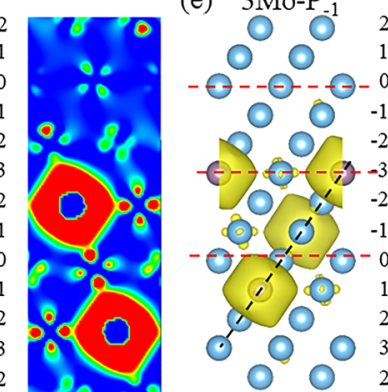

(f)

(f) $3 \mathrm{Mo}-\mathrm{P}_{3}$

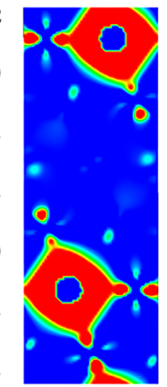

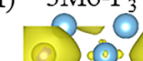
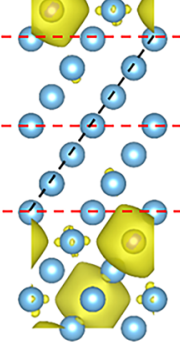

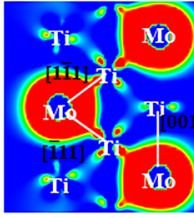

(110)

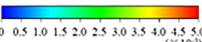

(g)

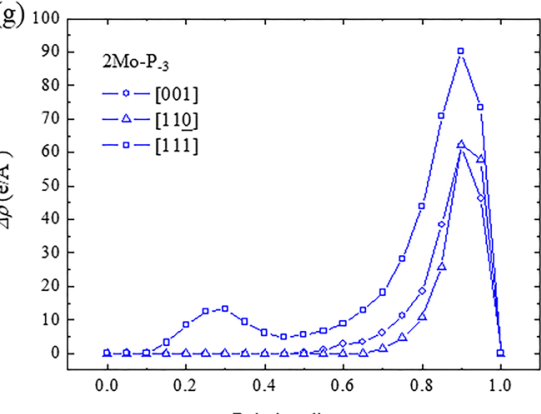

(h)
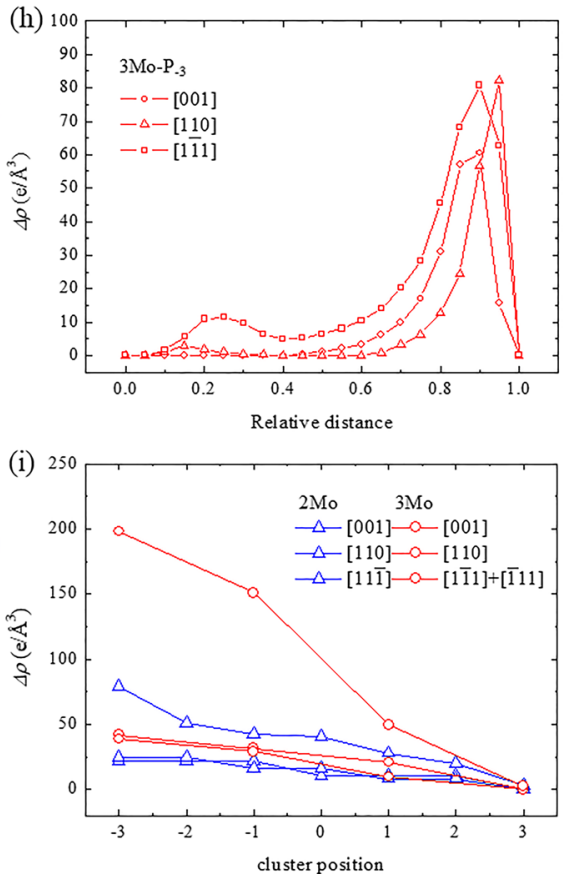

Figure 6. Charge density difference $(\Delta \rho)$ of the $\{110\}_{3}$ plane in the 2 Mo supercell with a linear cluster at $(\mathbf{a}) \mathrm{P}_{-3}$, (b) $\mathrm{P}_{0}$, and (c) $\mathrm{P}_{3}$ and in the 3 Mo supercell with a trigonal cluster at (d) $\mathrm{P}_{-3},(\mathbf{e}) \mathrm{P}_{-1}$, and (f) $\mathrm{P}_{3}$. The line profile of $\Delta \rho$ along the $<001>_{\beta},<011>_{\beta}$, and $<111>_{\beta}$ directions in the (g) 2 Mo supercell with a linear cluster at $\mathrm{P}_{-3}$, (h) 3 Mo supercell with a trigonal cluster at $\mathrm{P}_{-3}$, and (i) integral $\Delta \rho$ in terms of line profile as a function of cluster position.

$\left(G_{111}\right)$ was influenced by the charge density difference between Ti and Mo atoms along specific directions. Given the -Mo-Ti-Mo- cluster on two adjacent $(11 \overline{2})_{3}$ planes, the charge density difference $(\Delta \rho)$ within the region as denoted by the red dotted lines in Fig. 6 was calculated to evaluate the atomic bonding along the $[001]_{\beta},[110]_{\beta}$ and $[11 \overline{1}]_{\beta}$ directions, which hindered the shear of $(11 \overline{2})_{3}$ planes along the $[111]_{\beta}$ direction. The $\Delta \rho$ was indicated with $0.005 \mathrm{e}^{-} / \AA^{3}$ iso-charge surface in yellow. The $\Delta \rho$ in the $(1 \overline{1} 0)_{3}$ plane with a linear cluster (Fig. 6(a)) was large around Mo atoms and exhibited obvious values along the $[001]_{\beta},[110]_{\beta}$ and $[11 \overline{1}]_{3}$ directions, thereby suggesting that a strong Ti-Mo bonding was formed along these directions within the $(1 \overline{1} 0)_{3}$ plane. In the 3 Mo supercell, a $(110)_{\beta}$ plane with a trigonal structure as indicated by the black dotted line in Fig. 6(d) was used to clearly illustrate the charge density difference. In addition, the Ti-Mo bonding was particularly strong in the $\langle 111\rangle_{\beta}$ direction. To further evaluate the difference of $\Delta \rho$ along specific directions, the line profiles of $\Delta \rho$ were denoted by the white lines from the Ti atom to the Mo atom in Fig. 6(a,d), and the results are shown in Fig. 6(g,h). The $[001]_{\beta},[110]_{\beta}$, $[\overline{1} 11]_{3}$ and $[1 \overline{1} 1]_{3}$ directions in the 3 Mo supercell with a trigonal cluster were analyzed. The $\Delta \rho$ along the $<111>_{\beta}$ direction was the largest, whereas that along the $\langle 100\rangle_{\beta}$ direction was the smallest, thereby indicating that the Ti-Mo bonding was strongest along the $<111>$ direction, which was in agreement with the findings of Wang ${ }^{57}$. From an electronic basis, this result further revealed the rationality of the-Mo-Ti-Mo- linear and trigonal cluster structures, which was only analyzed based on cohesive energy in our previous work ${ }^{41}$. Similarly, the $\Delta \rho$ along specific directions was calculated when the linear cluster in Fig. 6(b,c) and trigonal cluster in Fig. 6(e,f) moved from $\mathrm{P}_{-3}$ to $\mathrm{P}_{3}$. For the line profiles such as those shown in Fig. $6(\mathrm{~g}, \mathrm{~h})$, the integral $\Delta \rho$ was obtained for each cluster position as shown in Fig. 6(i). The quantitative results indicated that the charge density difference along a specific direction in the $2 \mathrm{Mo}$ supercell was lower than that in the $3 \mathrm{Mo}$ supercell and decreased as the clusters moved from $\mathrm{P}_{-3}$ to $\mathrm{P}_{3}$. Therefore, the low charge density difference within the-Mo-Ti-Mo-cluster poor region 

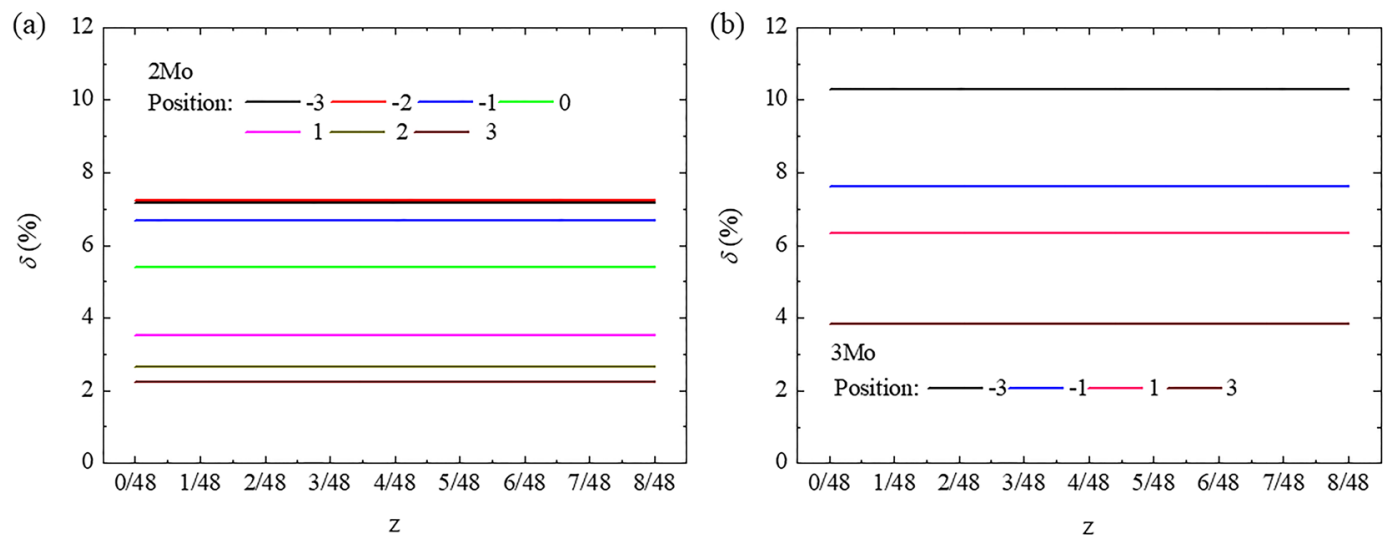

Figure 7. Change of lattice distortion $(\delta)$ along the $[11 \overline{2}]_{3}$ direction with collapse degree $(z)$ along the $[111]_{\beta}$ direction on the $(11 \overline{2})_{3}$ plane in (a) the 2 Mo supercell with a linear cluster and in (b) the 3 Mo supercell with a trigonal cluster at different positions.

indicated a reduction in atomic bonding or the softening of the shear modulus, which could benefit the $\omega$-phase formation.

Collapsed structure of the $\omega$-phase by stacking fault. Our previous theoretic calculations and experimental observations revealed that the $\omega$-phase structure changed from hexagonal to trigonal along with an increasing Mo content ${ }^{41}$. The experimental evidence also indicated that the $\omega$-phase with a trigonal structure was formed in the Mo-depleted region (Fig. 3). Although the aforementioned extrinsic and intrinsic factors were reasonable enough to explain the easy formation of the $\omega$-phase within the -Mo-Ti-Mo- cluster poor region, a direct correlation between the collapse degree of the $\omega$-phase and cluster structure was not clearly revealed. From the collapse mechanism of the $\omega$-phase, the stacking sequence of the $\{111\}_{3}$ plane in the bcc lattice changes from "ABCABCA" to "ABABA" along the $<111\rangle_{\beta}$ direction, whereas the structural evolution of the $\omega$-phase can be influenced by the stacking fault on the $\{112\}_{\beta}$ plane $^{34,42,43}$. These results also indicated that the stacking fault energy decreased along with the collapse degree and the collapsed structure was the most stable at the present minimum stacking fault energy (Figs. 1 and 2). On the basis of the constructed supercells comprising a cluster structure and stacking fault as shown in Fig. 1(b,c,e,f), both the lattice distortion and change density difference were used to examine the stacking fault energy and collapse degree of the $\omega$-phase. On the one hand, the lattice distortion along the $[11 \overline{2}]_{3}$ direction was not changed by the shear of atoms along the $[111]_{3}$ direction on the $(11 \overline{2})_{3}$ plane. For each cluster position in the $2 \mathrm{Mo}$ and 3 Mo supercells, the value of $\delta$ did not change along with collapse degree from 0 to $1 / 6$ as shown in Fig. 7, thereby suggesting that the changing tendency of stacking fault energy in Fig. 1(g,h) was not reasonably explained by the lattice distortion.

On the other hand, the stacking fault energy was affected by the charge density difference or atomic bonding, and the variation of stacking fault energy could be analyzed by the redistribution of change density, where some bonding may become either stronger or weaker ${ }^{58,59}$. Shang et al ${ }^{60}$ reported that the larger charge density difference of $\mathrm{Al}$ along the [11 20 ] direction led to higher stacking fault energy and shear strength along the [10 $\overline{1} 0]$ direction in Mg-based alloys. Kioussis et al ${ }^{6}{ }^{6}$ found that the variation of stacking fault energy in fcc metals resulted from the change of charge density in three directions across to the $\{111\}$ slip plane. Compared with the charge density difference without a collapse degree as shown in in Fig. 6, the values of integral $\Delta \rho$ for each cluster position in the $2 \mathrm{Mo}$ and $3 \mathrm{Mo}$ supercells were calculated at different collapse degrees along specific directions. Here, the difference of $\Delta \rho(d \Delta \rho)$ with and without collapse degree was calculated to describe the redistribution of charge density as shown in Fig. 8. In the 2Mo supercell, the $d \Delta \rho$ along the $<100>_{\beta}$ direction increased at each cluster position along with an increasing collapse degree but did not show any changes at $\mathrm{P}_{3}$ as shown in Fig. 8(a). As shown in Fig. 8(b), the $d \Delta \rho$ along the $\langle 110\rangle_{\beta}$ direction did not obviously change along with collapse degree at all cluster positions. By contrast, the $d \Delta \rho$ along the $\langle 111\rangle_{\beta}$ direction exhibited a decreasing trend at each cluster position with an increasing the collapse degree, except at the position of $\mathrm{P}_{3}$ as shown in Fig. 8(c). Although the trends of $d \Delta \rho$ (Fig. 8(d-f)) were similar to those recorded in the 2 Mo supercell, larger variations in the values of $d \Delta \rho$ were observed along the $\langle 100\rangle_{\beta}$ and $\langle 111\rangle_{\beta}$ directions in the 3 Mo supercell. To synthetically examine the effect of charge density difference on stacking fault energy, the total values of $\Delta \rho$ and $d \Delta \rho$ along three directions were calculated at different collapse degrees as shown in Fig. 9. The total $\Delta \rho$ decreased for each collapse degree when the cluster structure moved from $\mathrm{P}_{-3}$ to $\mathrm{P}_{3}$ and decreased along with an increasing collapse degree at each cluster position, except at $\mathrm{P}_{3}$ (where no obvious changes were observed) in 2Mo and $3 \mathrm{Mo}$ supercells and at $\mathrm{P}_{-3}$ (where an increasing trend was reported) in the $3 \mathrm{Mo}$ supercell. Moreover, a minimum charge density difference was observed at a collapse degree of less than or equal to $1 / 6$ when the cluster position was near to or far from the stacking fault. The minimum charge density difference corresponded to the zero collapse degree (bcc lattice) in the $3 \mathrm{Mo}$ supercell with the cluster at $\mathrm{P}_{-3}$. The variations in charge density difference along with cluster position and collapse degree agreed with the trends of stacking fault energy and indicated the collapsed structural evolution of $\omega$-phase, even when the overestimated and underestimated values were considered. 

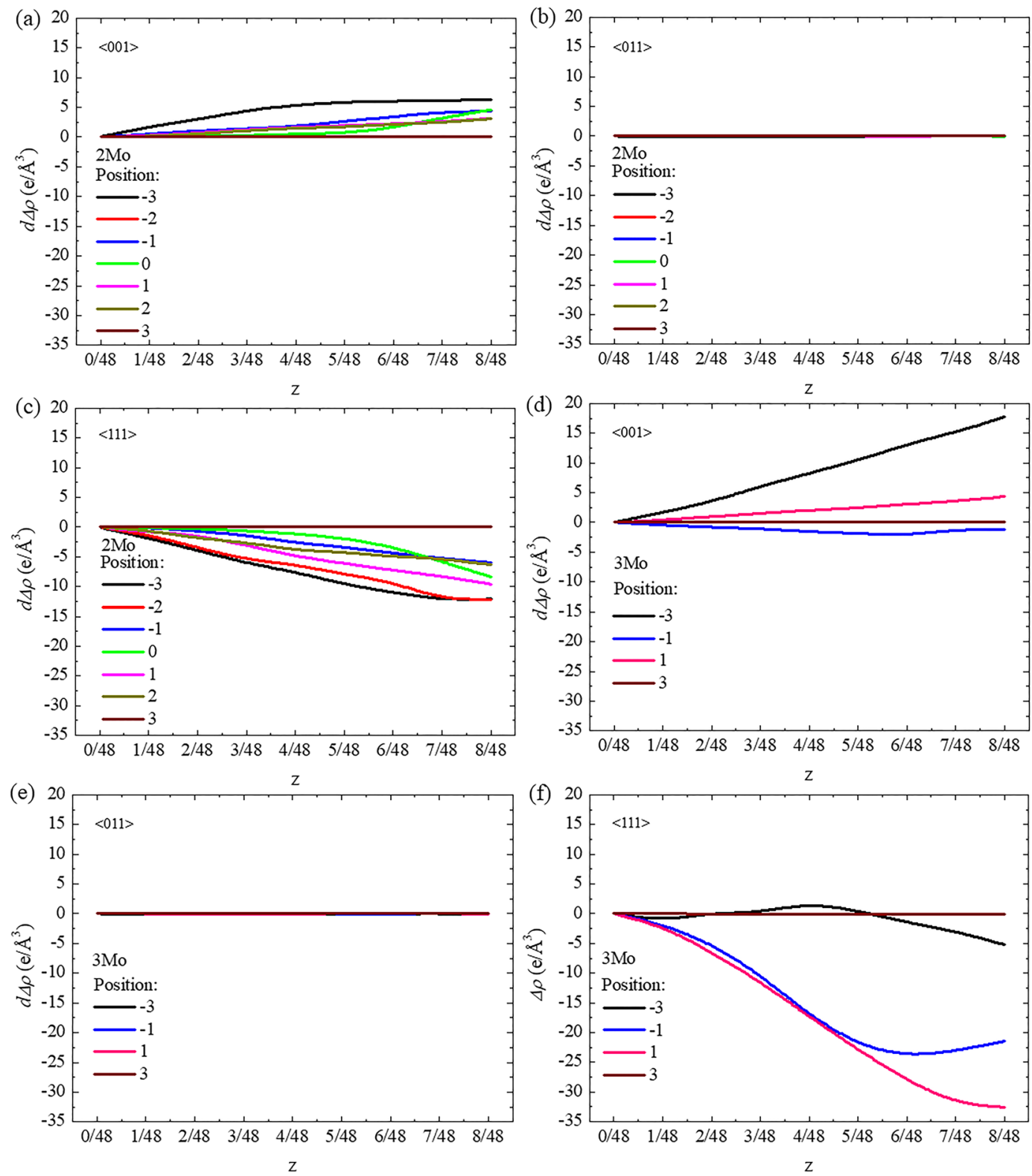

Figure 8. Difference of charge density difference $(d \Delta \rho)$ as a function of collapse degree $(\mathrm{z})$ on the $\{110\}_{\beta}$ plane along the $(\mathbf{a})<001>_{\beta}$, (b) $<011>_{\beta}$, and (c) $<111>_{\beta}$ directions in the 2 Mo supercell and along the (d) $<001>_{\beta}$, (e) $<011>_{\beta}$, and $(\mathbf{f})<111>_{\beta}$ directions in the 3 Mo supercell.

On the basis of the theoretic calculations of stacking fault energy and charge density difference, in the Mo-depleted region, the $\omega$-phase with a hexagonal structure was easily formed, while the experimental evidence confirmed the formation of a trigonal $\omega$-phase due to the compositional instability under the water-quenching condition, that is, a metastable "frozen" state of Mo atoms, thereby leading to the present minimum stacking fault energy (minimum charge density difference) with a partial collapse degree (Figs. 1 and 9). If the Mo atoms sufficiently moved out of the Mo-depleted region, then the collapse degree of the $\omega$-phase would increase up to $1 / 6$, as reported in ref. ${ }^{10,33}$. However, the formation of an isothermal $\omega$-phase was not the focus of this work. The local instability of the composition is known to be present in quenched $\beta$-type titanium alloys, which often leads to local structural instability ${ }^{10,34,36,62,63}$. In addition, the nano-scale compositional partitioning resulted in continuous changes in the solute content between the depleted and enriched regions as shown in Figs. 3 and 4. Therefore, the structural instability of the $\omega$-phase changed continuously due to compositional instability. Consequently, the structural evolution of the $\omega$-phase from hexagonal to trigonal in metastable $\beta$-type Ti-Mo alloys was schematically drawn along with Mo content and collapse degree in Fig. 10. The concurrent compositional and structural instabilities of the $\omega$-phase was attributed to the coupling effect of the cluster structure with stacking fault.

In conclusion, the -Mo-Ti-Mo- cluster structures should occupy positions far from the stacking fault in the constructed supercells with a linear cluster along the $\langle 111\rangle_{\beta}$ direction and a trigonal cluster along the $\langle 111\rangle_{\beta}$ and $<001\rangle_{\beta}$ directions. The stable structure of the $\omega$-phase with different collapse degrees on the $\{112\}_{\beta}$ plane 

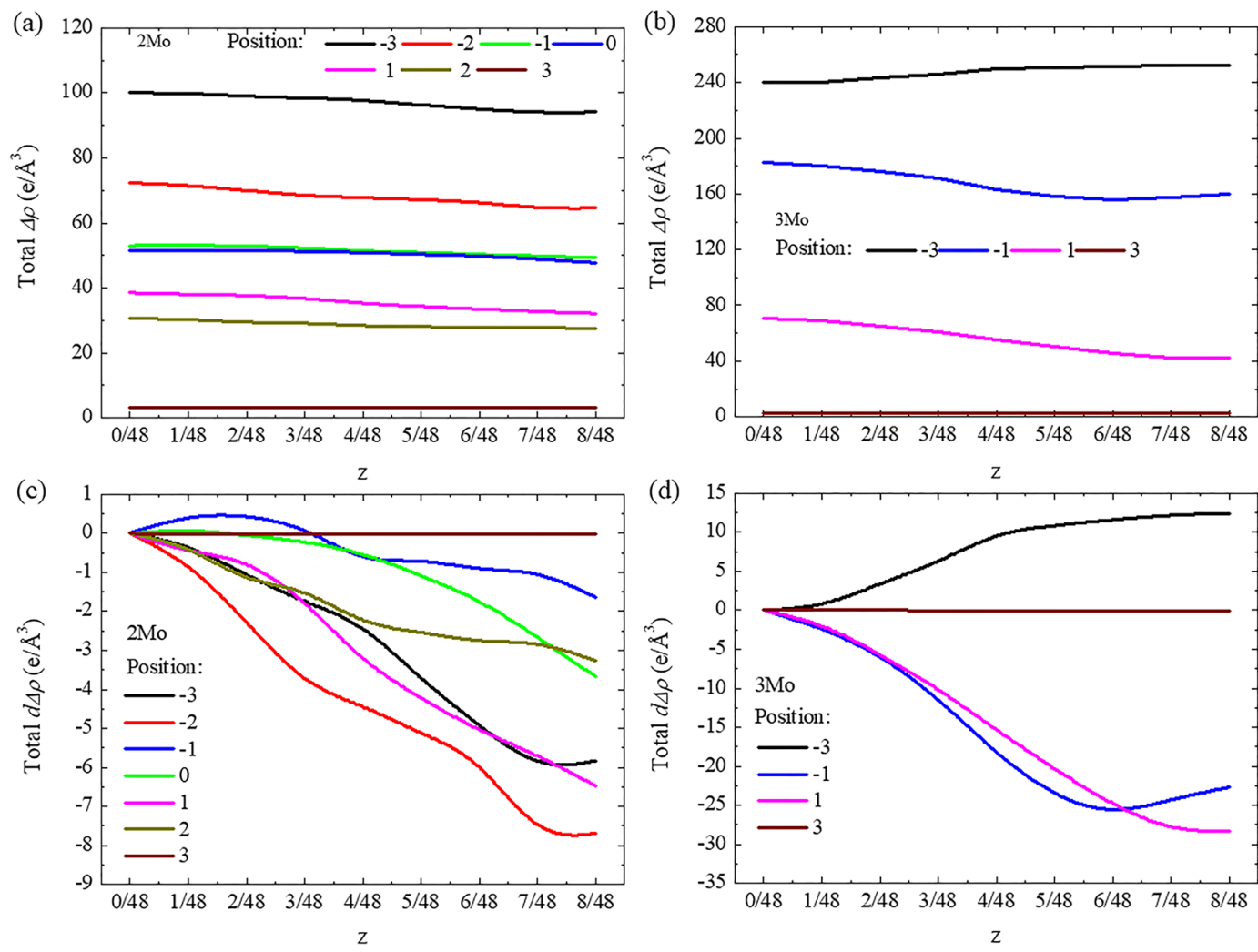

Figure 9. Total charge density difference (total $\Delta \rho$ ) in (a) the $2 \mathrm{Mo}$ supercell and (b) the $3 \mathrm{Mo}$ supercell, and total difference of charge density difference (total $d \Delta \rho$ ) in (c) the 2 Mo supercell and (d) the 3 Mo containing $<001>_{\beta}$, $<011>_{\beta}$ and $<111>_{\beta}$ directions on the $\{110\}_{\beta}$ plane as a function of collapse degree $(z)$.

along the $<111>_{\beta}$ direction strongly depended on the present minimum stacking fault energy and the formation energy of cluster structures. The $\omega$-phase with a partially collapsed structure was prone to form in the nano-scale Mo-depleted region due to compositional partitioning under a rapid cooling condition. The structural instability of the $\omega$-phase continuously changed along with compositional instability from the Mo-enriched region to the Mo-depleted region due to the metastable "frozen" state of Mo atoms. Within the cluster poor region, the lattice distortion along the $\langle 112\rangle_{\beta}$ direction on the $\{112\}_{\beta}$ plane decreased, thereby leading to the low energy barrier in the collapse pathway. Moreover, the charge density difference corresponding to the atomic bonding along the $<111\rangle_{\beta},<011>_{\beta}$ and $<001>_{\beta}$ directions on the $(110)_{\beta}$ plane was reduced, thereby softening the shear modulus $\left(G_{111}\right)$ along the $\left.<111\right\rangle_{\beta}$ direction on the $\{112\}_{\beta}$ plane, which benefited the $\omega$-phase formation. The redistribution of charge density exhibited an obvious anisotropy among the $\langle 111\rangle_{\beta},\langle 011\rangle_{\beta}$, and $\langle 001\rangle_{\beta}$ directions with different collapse degrees, and the changing tendency of total charge density difference on the $(110)_{3}$ plane with cluster structure and collapse degree was in accordance with the stacking fault energy and corresponded to the collapsed structural evolution of the $\omega$-phase from trigonal to hexagonal.

\section{Methods}

Calculation methodology. The calculations were carried out based on density functional theory and by using the Vienna Ab-initio Simulation Package code ${ }^{64}$ and the projector augmented wave method for investigating the core-valence electrons interaction ${ }^{65}$. The exchange correlation potential was measured via generalized gradient approximation and expressed in Perdew-Burke- Ernzerhof form ${ }^{66}$. The plane wave cut-off energy was set at $400 \mathrm{eV}$. Relaxation was stopped when the energy reached $10^{-3} \mathrm{eV}$ convergence. The Monkhorst-Pack mesh for this system was $9 \times 11 \times 1$. Supercells comprising 24 atoms were constructed with $12(11 \overline{2})_{3}$ planes and 2 and 3 Mo atoms (Ti-15Mo or Ti-22Mo alloys, respectively). According to the collapse mechanism of the $\omega$-phase, the two adjacent $(11 \overline{2})_{\beta}$ planes shifted toward each other via $z a_{0}[111]_{\beta}$, where $z$ is the collapse degree from 0 to $1 / 6$ with a step of $1 / 48$, and $a_{0}$ is the lattice parameter of the $\beta$-phase. The -Mo-Ti-Mo- linear unit along the [ $11 \overline{1}_{\beta}$ direction is the most feasible configuration of two Mo atoms ${ }^{40}$ and has seven positions in the supercell. For three Mo atoms, a triangle cluster with three linear units along different directions (i.e., $[1 \overline{1} 1]_{3},[\overline{1} 11]_{\beta}$ and $[001]_{\beta}$ ) is the most feasible configuration ${ }^{40}$ with four positions in the supercell.

Stacking fault energy $\left(\gamma_{S F}^{z}\right)$ was calculated as follows in terms of the constructed supercells with cluster structure and stacking fault ${ }^{48}$ : 


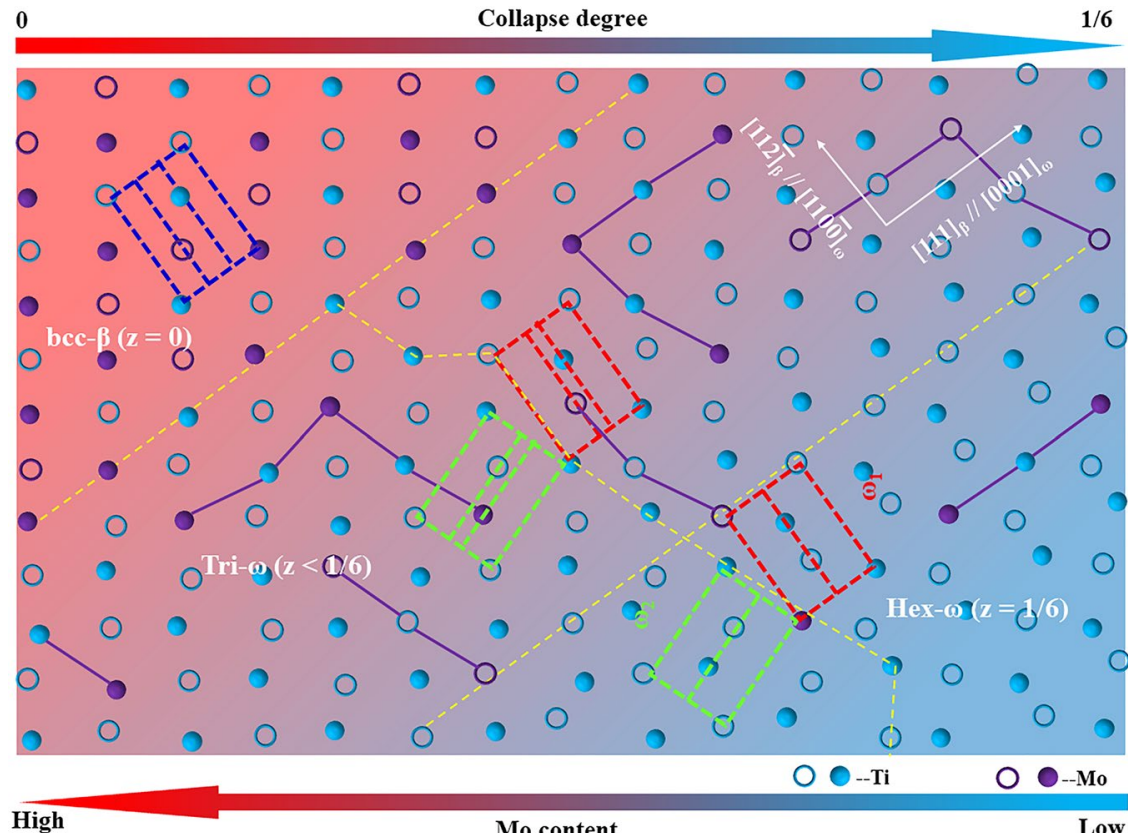

Figure 10. Schematic diagram of the structural evolution of the $\omega$-phase $\left(\omega_{1}\right.$ - and $\omega_{2}$-variants: red and green motifs) from hexagonal to trigonal in the $\beta$-matrix (blue motif) of metastable Ti-Mo alloys with an increasing Mo content and decreasing collapse degree. The open and solid circles were located at two adjacent $(1 \overline{1} 0)_{3}$ planes.

$$
\gamma_{S F}^{z}=\frac{E_{Z}-E_{0}}{A}
$$

where $E_{z}$ and $E_{0}$ are the total energy of a supercell with a collapse degree and the total energy of a perfect bcc supercell, respectively, and $A$ is the area of the fault plane that is a cross product of supercell lattice vectors $a_{0}[1 \overline{1} 0]$ ${ }_{\beta}$ and $a_{0}[111]_{\beta}$. To further evaluate the stability of an Mo atom occupying a certain position in the supercell, the formation energy $\left(E_{F}\right)$ was calculated as

$$
E_{F}=E_{z}-n E_{M o}^{b c c}-(24-n) E_{T i}^{h c p}-A E_{S F}^{T i}
$$

where $E_{T i}^{h c p}$ and $E_{M o}^{b c c}$ are the energies per atom of bulk Ti in the hexagonal close-packed structure and that of Mo in the bcc structure, respectively, $n$ is the number of Mo atoms in each supercell, and $E_{S F}^{T i}$ is the stacking fault energy without Mo atoms. Following this definition, a lower formation energy corresponds to a more stable Mo atom at a certain position. The formation energy was calculated only in the structure with the minimum stacking fault energy.

The lattice distortion $(\delta)$ of each supercell was calculated due to the different radiuses of the Mo and Ti atoms, and the quantitative value of the $(1 \overline{1} 0)_{\beta}$ plane along the $[11 \overline{2}]_{\beta}$ direction was computed as

$$
D=\left(x^{\prime}-x\right) \cos \alpha_{3} \cos \alpha_{1}+\left(y^{\prime}-y\right) \cos \alpha_{2}+\left(z^{\prime}-z\right) \cos \alpha_{3},
$$

where $x^{\prime}, y^{\prime}, z^{\prime}$ and $x, y, z$ are the fractional coordinates of atoms with distortion and non-distortion, respectively,

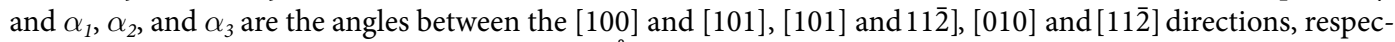
tively. The charge density difference $\left(\Delta \rho\right.$ in $\left.\mathrm{e}^{-} / \AA^{3}\right)$ of each supercell was calculated as follows to evaluate the atomic bonding ${ }^{11}$ :

$$
\Delta \rho=\rho_{T i-M o}-\rho_{T i},
$$

where $\rho_{T i-M o}$ is the charge density of the supercell containing Ti and Mo atoms, and $\rho_{T i}$ is the charge density of the supercell with Mo replaced by Ti atoms. The charge density difference on the $(1 \overline{1} 0)_{3}$ plane along the $<001>_{\beta}$, $<011\rangle_{\beta}$ and $<111>_{\beta}$ directions was quantitatively evaluated.

Material preparation and microstructural observation. Ti-15Mo alloy was prepared via cold crucible levitation melting. The ingot weighed approximately $1 \mathrm{~kg}$ and was homogenized at $1273 \mathrm{~K}$ for $3.6 \mathrm{ks}$, hot forged into a block at $1273 \mathrm{~K}$, hot rolled into a plate with dimensions of $290 \mathrm{~mm}(l) \times 50 \mathrm{~mm}(w) \times 10 \mathrm{~mm}(t)$ at $1173 \mathrm{~K}$, and eventually air cooled. After cutting into $10 \mathrm{~mm}(l) \times 10 \mathrm{~mm}(w) \times 5 \mathrm{~mm}(t)$, the plate was solution treated for $3.6 \mathrm{ks}$ at $1173 \mathrm{~K}$ and underwent water quenching. Our previous works ${ }^{67,68}$ reported that the chemical 
compositions of Ti-Mo alloys were controlled well by using this melting method and that each alloy had an oxygen content of approximately $0.1 \mathrm{wt} . \%$ with small amounts of nitrogen and carbon.

The sample of $10 \mathrm{~mm}(l) \times 10 \mathrm{~mm}(w) \times 1 \mathrm{~mm}(t)$ was cut from the solution-treated plate via wire cut electrical discharge machining and then mechanically ground to $0.1 \mu \mathrm{m}$ in thickness. Subsequently, the foils for microstructural characterization were thinned via dimple grinding (Gatan 656 Dimple Grinder) and ion milling (Gatan PIPS II 695). TEM and atomic resolution HAADF-STEM observations were carried out by using a $300 \mathrm{kV}$ probe corrected electron microscope (FEI Titan G2). HAADF images were captured by using a probe with a convergence semi-angle of $21.4 \mathrm{mrad}$ by setting the camera length at $100 \mathrm{~mm}$. The inner and outer detection angles were $76 \mathrm{mrad}$ and $200 \mathrm{mrad}$, respectively. To illustrate the structural features at the atomic level, Fourier filtering processing was performed by using a digital micrograph software to remove noisy background from raw images, and the local structural information was analyzed by Fast Fourier Transform (FFT) patterns.

Received: 7 February 2020; Accepted: 8 April 2020;

Published online: 26 May 2020

\section{References}

1. Frost, P. D., Parris, W. M., Hirsh, L. L., Diog, J. R. \& Schwartz, C. M. Isothermal transformation of titanium-chromium alloys, Trans. Am. Soc. Met 46, 231-256 (1954).

2. Banerjee, S. \& Mukhopadhyay, P. Transformations Related to Omega Structures, In: R.W. Cahn (Ed.), Phase Transformations: Examples from Titanium and Zirconium Alloys, Elsevier, Great Britain,474-550, (2007).

3. Sikka, S. K., Vohra, Y. K. \& Chidambaram, R. Omega phase in materials. Prog. Mater. Sci. 27, 245-310 (1982).

4. Banerjee, S. \& Mukhopadhyay, P. Phase transformations: examples from titanium and zirconium alloys. Oxford: Pergamon Press; 2004.

5. Banerjee, D. \& Williams, J. C. Perspectives on titanium science and technology. Acta Mater. 61, 844-879 (2013).

6. Liu, H., Niinomi, M., Nakai, M. \& Cho, K. Athermal and deformation-induced $\omega$-phase transformations in biomedical beta-type alloy Ti-9Cr-0.2O. Acta Mater. 106, 162-170 (2016).

7. Liu, H., Niinomi, M., Nakai, M., Cho, K. \& Fujii, H. Deformation-induced $\omega$-phase transformation in a $\beta$-type titanium alloy during tensile deformation. Scripta Mater. 130, 27-31 (2017).

8. Tane, M. et al. $\omega$ Transformation in cold-worked Ti-Nb-Ta-Zr-O alloys with low body centered cubic phase stability and its correlation with their elastic properties. Acta Mater. 61, 139-150 (2013).

9. Mantri, S. A. et al. Change in the deformation mode resulting from beta-omega compositional partitioning in a Ti-Mo alloy: Room versus elevated temperature. Scripta Mater. 130, 69-73 (2017).

10. Devaraj, A. et al. Experimental evidence of concurrent compositional and structural instabilities leading to $\omega$ precipitation in titanium-molybdenum alloys. Acta Mater. 60, 596-609 (2012).

11. Choudhuri, D. et al. Coupled experimental and computational investigation of omega phase evolution in a high misfit titaniumvanadium alloy. Acta Mater. 130, 215-228 (2017).

12. Ahmed, M., Wexler, D., Casillas, G., Ivasishin, O. M. \& Pereloma, E. V. The influence of $\beta$ phase stability on deformation mode and compressive mechanical properties of Ti-10V-3Fe-3Al alloy. Acta Mater. 84, 124-135 (2015).

13. Zhang, J., Tasan, C. C., Lai, M. J., Dippel, A. C. \& Raabe, D. Complexion-mediated martensitic phase transformation in Titanium. Nat. Commun. 8, 14210 1-8 (2017).

14. M. Niinomi, et al, Influence of oxygen on omega phase stability in the Ti-29Nb-13Ta-4.6Zr alloy, Scripta Mater. 123, 144-148 (2016).

15. Castany, P., Yang, Y., Bertrand, E. \& Gloriant, T. Reversion of a parent $\{130\}<310>$ alpha' martensitic twinning system at the origin of $\{332\}<113>$ beta twins observed in metastable beta titanium alloys. Phys. Rev. Lett. 117(24), 245501 1-6 (2016).

16. Zhao, X. F., Niinomi, M., Nakai, M. \& Hieda, J. Beta type Ti-Mo alloys with changeable Young's modulus for spinal fixation applications. Acta Biomater. 8, 1990-1997 (2012).

17. Lai, M. J., Li, T. \& Raabe, D. $\omega$ phase acts as a switch between dislocation channeling and joint twinning- and transformationinduced plasticity in a metastable $\beta$ titanium alloy. Acta Mater. 151, 67-77 (2018).

18. Ho, J. C. \& Collings, E. W. The Influence of $\omega$-phase on the superconductivity of Ti-Mo alloys. Phys. Lett. A 29, 206-207 (1969).

19. Fontaine, D. D., Buck, O. \& Monte Carlo, A. simulation of the omega phase transformation. Philos. Mag. 27, 967-983 (1973).

20. Fontaine, D. D. Simple models for the omega phase transformation, Metall. Trans. A 19, 169-175 (1988).

21. Lin, C., Yin, G. L., Zhang, A. M., Zhao, Y. Q. \& Li, Q. C. Simple models to account for the formation and decomposition of athermal $\omega$ phase in titanium alloys. Scripta Mater. 117, 28-31 (2016).

22. Fontaine, D. D., Paton, N. E. \& Williams, J. C. Omega phase transformation in titanium alloys as an example of displacement controlled reactions. Acta Metall. 19, 1153-1162 (1971).

23. Ho, K. M., Fu, C. L. \& Harmon, B. N. Microscopic analysis of interatomic forces in transition metals with lattice distortions. Phys. Rev. B 28, 6687-6694 (1983).

24. Lin, C., Yi, G. L., Zhang, A. M., Zhao, Y. Q. \& Li, Q. C. Simple models to account for the formation and decomposition of athermal $\omega$ phase in titanium alloys. Scripta Mater. 117, 28-31 (2016).

25. Wang, H. L. et al. Stabilizing the body centered cubic crystal in titanium alloys by a nano-scale concentration modulation. J. Alloy Compd. 700, 155-158 (2017).

26. Grad, G. B., Benites, G. M. \& Aurelio, G. A. Fernandez Guillermet, Bond-length analysis of the omega structure in Ti, Zr, Hf and their alloys: experimental data, new correlations and implications for chemical bonding models. Mater. Sci. Eng. A 273-275, 175-180 (1999).

27. Benites, G. M., Aurelio, G., Fernandez Guillermet, A., Cuello, G. J. \& Bermejo, F. J. Atomic ordering and systematics of bonding lengths in the Ti-V omega phase: a neutron diffraction study. J. Alloy Compd. 284, 251-255 (1999).

28. Benites, G. M., Fernandez Guillermet, A., Cuello, G. J. \& Campo, J. Structural properties of metastable phases in Zr-Nb alloys I. Neutron diffraction study and analysis of lattice parameters. J. Alloy Compd. 299, 183-188 (2000).

29. Benites, G. M. \& Fernandez Guillermet, A. Structural properties of metastable phases in Zr-Nb alloys II. Systematics of the atomic volumes and interatomic distances. J. Alloy Compd. 302, 192-198 (2000).

30. Cuello, G. J., Aurelio, G., Fernandez Guillermet, A., Benites, G. M. \& Campo, J. Bonding-induced atomic ordering in the athermal $\omega$ phase: neutron diffraction test and consequences upon models of the bond-length systematics in alloys. Scripta Mater. 44, 2821-2825 (2001).

31. Aurelio, G., Fernandez Guillermet, A., Cuello, G. J. \& Campo, J. Structural properties of metastable phases in Zr-Nb alloys III. The athermal $\omega$ phase. J. Alloy Compd. 335, 132-138 (2002).

32. Hickman, B. S. The formation of omega phase in titanium and zirconium alloys: a review. J. Mater. Sci. 4, 554-563 (1969).

33. Nag, S. et al. Novel mixed-mode phase transition involving a composition-dependent displacive component. Phys. Rev. Lett. 106, 1-4 (2011). 
34. Lai, M. J. et al. Origin of shear induced $\beta$ to $\omega$ transition in Ti-Nb based alloys. Acta Mater. 92, 55-63 (2015).

35. Niu, J. G., Ping, D. H., Ohno, T. \& Geng, W. T. Suppression effect of oxygen on the $\beta$ to $\omega$ transformation in a $\beta$-type Ti alloy: insights from first-principles. Modelling Simul. Mater. Sci. Eng. 22, 1-9 (2014).

36. Li, Z., Zhou, Z. H., Wang, H. B., Li, S. L. \& Zhao, Z. First-principles study on stability, and growth strategies of small AlnZr (N = 1-9) clusters. J. Cryst. Growth 449, 22-26 (2016).

37. Lu, J., Wei, S. H., Zhang, Y. Y., Hua, D. Y. \& Duan, X. M. Geometric, electronic and magnetic properties of Au-N, Aun-1Pt and Aun2 Pt2 (N = 2-9) clusters: a first-principles study. Comput. Theor. Chem. 1090, 157-164 (2016).

38. Rodriguez-Kessler, P. L. \& Rodriguez-Dominguez, A. R. Structures and electronic properties of TinV ( $\mathrm{N}=1-16)$ clusters: Firstprinciples calculations. J. Phys. Chem. A 120, 2401-2407 (2016).

39. Otani, N. et al. Theoretical investigation of solid solution states of Ti1-xVxH2. Acta Mater. 134, 274-282 (2017).

40. Li, M. J. et al. First-principles study of phase stability and elastic property in metastable Ti-Mo alloys with cluster structure. Mol. Simulat. 45, 26-34 (2019).

41. Li, M. J., Min, X. H., Yao, K. \& Ye, F. Novel insight into the formation of $\alpha^{\prime \prime}$-martensite and $\omega$-phase with cluster structure in metastable Ti-Mo alloys. Acta Mater. 164, 322-333 (2019).

42. Jackson, A. G. On the relationship between omega phase and twinning bcc structure. Scripta Metal 22, 313-316 (1988).

43. Wu, S. Q. et al. $\{112\}<111>$ Twinning during $\omega$ to body-centered cubic transition. Acta Mater. 62, 122-128 (2014).

44. Chen, B. \& Sun, W. Omega transitional structure associated with $\{112\}<111>$ deformation twinning in a metastable beta Ti-Nb alloy, revealed by atomic resolution high-angle annular dark-field scanning transmission electron microscopy. J. Alloy Compd. 766, 123-130 (2018)

45. Talonen, J. \& Hanninen, H. Formation of shear bands and strain-induced martensite during plastic deformation of metastable austenitic stainless steels. Acta Mater. 55, 6108-6118 (2007).

46. Lee, T. H., Shin, E., Oh, C. S., Ha, H. Y. \& Kim, S. J. Correlation between stacking fault energy and deformation microstructure in high-interstitial-alloyed austenitic steels. Acta Mater. 58, 3173-3186 (2010).

47. Medvedeva, N. I., Park, M. S., Van Aken, D. C. \& Medvedeva, J. E. First-Principles study of Mn, Al and C distribution and their effect on stacking fault energies in Fcc Fe. J. Alloy Compd. 582, 475-482 (2014).

48. Abbasi, A., Dick, A., Hickel, T. \& Neugebauer, J. First-principles investigation of the effect of carbon on the stacking fault energy of Fe-C Alloys. Acta Mater. 59, 3041-3048 (2011)

49. Zhang, Z. J. et al. Nanoscale origins of the damage tolerance of the high-entropy alloy CrMnFeCoNi. Nat. Commun. 6, 10143 1-6 (2015).

50. Wang, L. H. et al. New twinning route in face-centered cubic nanocrystalline metals. Nat. Commun. 8, 2142 1-7 (2017).

51. Huang, S. et al. Twinning in metastable high-entropy alloys. Nat. Commun. 9, 2381 1-7 (2018).

52. Niu, C. N., LaRosa, C. R., Miao, J. S., Mills, M. J. \& Ghazisaeidi, M. Magnetically-driven phase transformation strengthening in high entropy alloys. Nat. Commun. 9, 1363 1-9 (2018).

53. Niu, J. G. \& Geng, W. T. Oxygen-induced lattice distortion in $\beta-\mathrm{Ti}_{3} \mathrm{Nb}$ and its suppression effect on $\beta$ to $\alpha^{\prime \prime}$ transformation. Acta Mater. 81, 194-203 (2014).

54. Jeong, H. W., Yoo, Y. S., Lee, Y. T. \& Park, J. K. Elastic softening behavior of Ti-Nb single crystal near martensitic transformation temperature. J. Appl. Phys. 108, 1-6 (2010).

55. Tane, M. et al. Low Young's modulus of Ti-Nb-Ta-Zr alloys caused by softening in shear moduli $\mathrm{c}^{\prime}$ and $\mathrm{c}_{44}$ near lower limit of bodycentered cubic phase stability. Acta Mater. 58, 6790-6798 (2010).

56. Gao, M. C. et al. Phase stability and elastic properties of Cr-V alloys. J. Phys.: Condens. Matter 25, 1-15. (2013)

57. Wang, W. Y. et al. Insight into solid-solution strengthened bulk and stacking faults properties in Ti alloys: a comprehensive firstprinciples study. J. Mater. Sci. 53, 7493-7505 (2018).

58. Ogata, S., Li, J. \& Yip, S. Ideal Pure Shear Strength of Aluminum and Copper. Science 298, 807-811 (2002).

59. Qi, Y. \& Mishra, R. K. Ab initio study of the effect of solute atoms on the stacking fault energy in aluminum. Phys. Rev. B. 75, 1-5 (2007).

60. Shang, S. L. et al. Generalized stacking fault energy, ideal strength and twinnability of dilute Mg-based alloys: A first-principles study of shear deformation. Acta Mater. 67, 168-180 (2014).

61. Kioussis, N., Herbranson, M. \& Collins, E. Topology of Electronic Charge Density and Energetics of Planar Faults in fcc Metals. Phys. Rev. Lett. 88, 1-4 (2002).

62. Hao, Y. L. et al. Superelasticity and tunable thermal expansion across a wide temperature range. J. Mater. Sci. Tech 32, 705-709 (2016).

63. Ng, H. P. et al. Phase separation and formation of omega phase in the beta matrix of a Ti-V-Cu alloy. Acta Mater. 59, 2981-2991 (2011).

64. Kresse, D. \& Furthmüler, J. Efficient iterative schemes for ab initio total-energy calculations using a plane-wave basis set. Phys. Rev. $B$ 54, 11169-11186 (1996).

65. Kresse, G. \& Joubert, D. From ultrasoft pseudopotentials to the projector augmented-wave method. Phys. Rev. B 59, 1758-1775 (1999).

66. Perdew, J. P., Burke, K. \& Ernzerhof, M. Generalized gradient approximation made simple. Phys. Rev. Lett. 77, 3865-3868 (1996).

67. Min, X. H., Emura, S., Nishimura, T., Tsuchiya, K. \& Tsuzaki, K. Microstructure, tensile deformation mode and crevice corrosion resistance in Ti-10Mo-xFe alloys. Mater. Sci. Eng. A. 527, 5499-5506 (2010).

68. Min, X. H. et al. Effect of oxygen content on deformation mode and corrosion behavior in $\beta$-type Ti-Mo alloy. Mater. Sci. Eng. A. 684, 534-541 (2017).

\section{Acknowledgements}

This work was financially supported by the National Key Research and Development Program of China, China (Grant No. 2016YFB1100103) and the National Natural Science Foundation of China, China (Grant No. 51471040). The authors were deeply grateful to Professor Koichi Tsuchiya and Principal Researcher Satoshi Emura of National Institute for Materials Science in Japan for their supports.

\section{Author contributions}

X.H. Min designed the project and guided the research. M.J. Li performed the calculations and experiments. Both authors participated in the discussion of the results and the writing of the manuscript.

\section{Competing interests}

The authors declare no competing interests. 


\section{Additional information}

Correspondence and requests for materials should be addressed to X.M.

Reprints and permissions information is available at www.nature.com/reprints.

Publisher's note Springer Nature remains neutral with regard to jurisdictional claims in published maps and institutional affiliations.

(c) (i) Open Access This article is licensed under a Creative Commons Attribution 4.0 International License, which permits use, sharing, adaptation, distribution and reproduction in any medium or format, as long as you give appropriate credit to the original author(s) and the source, provide a link to the Creative Commons license, and indicate if changes were made. The images or other third party material in this article are included in the article's Creative Commons license, unless indicated otherwise in a credit line to the material. If material is not included in the article's Creative Commons license and your intended use is not permitted by statutory regulation or exceeds the permitted use, you will need to obtain permission directly from the copyright holder. To view a copy of this license, visit http://creativecommons.org/licenses/by/4.0/.

(C) The Author(s) 2020 